This item was submitted to Loughborough's Research Repository by the author.

Items in Figshare are protected by copyright, with all rights reserved, unless otherwise indicated.

\title{
Large eddy simulation of a controlled diffusion compressor cascade
}

PLEASE CITE THE PUBLISHED VERSION

http://dx.doi.org/10.1007/s10494-010-9314-z

PUBLISHER

(C) Springer

VERSION

AM (Accepted Manuscript)

LICENCE

CC BY-NC-ND 4.0

REPOSITORY RECORD

McMullan, W.A., and Gary J. Page. 2012. "Large Eddy Simulation of a Controlled Diffusion Compressor Cascade". figshare. https://hdl.handle.net/2134/9497. 
This item was submitted to Loughborough's Institutional Repository (https://dspace.lboro.ac.uk/) by the author and is made available under the following Creative Commons Licence conditions.

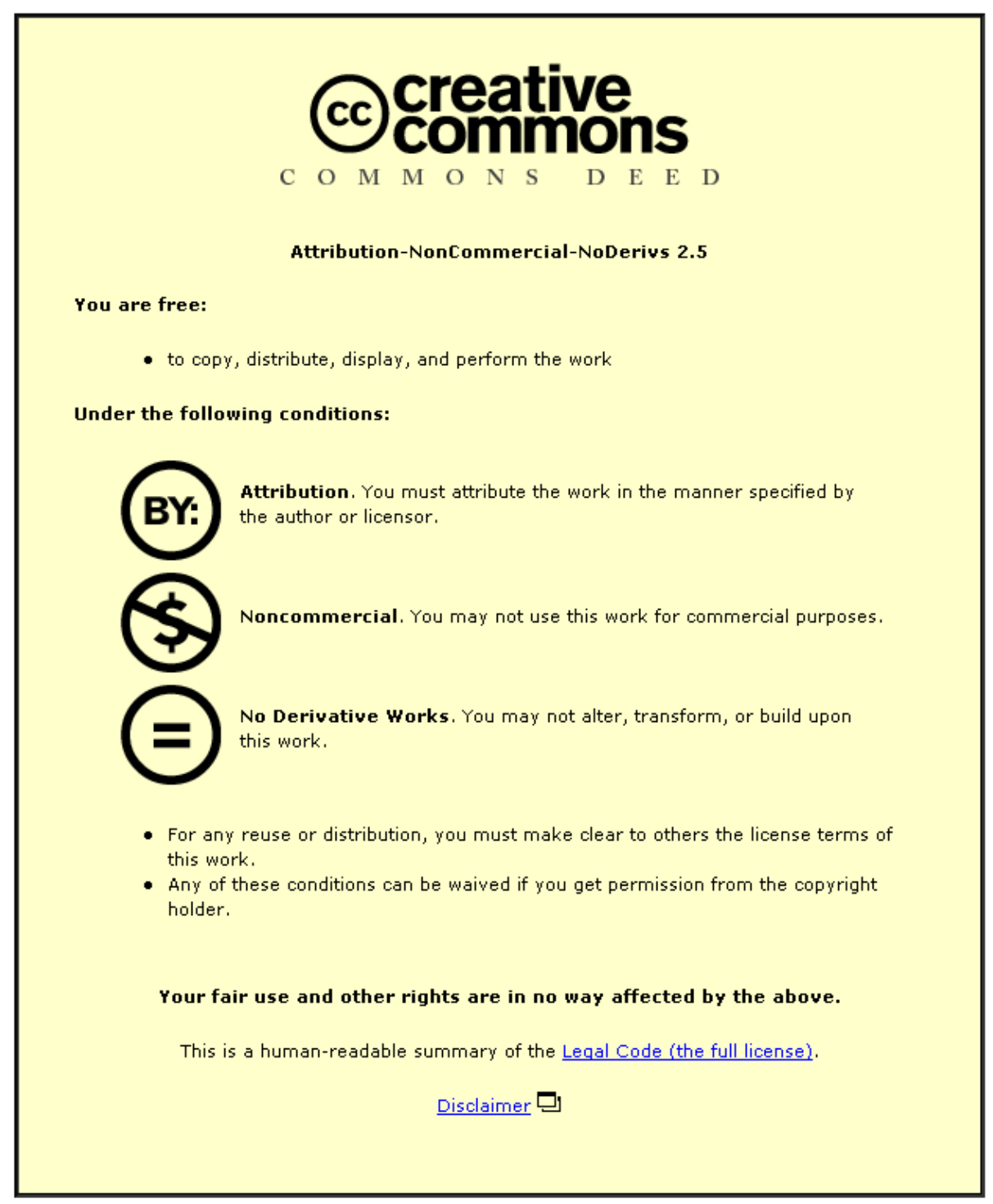

For the full text of this licence, please go to: http://creativecommons.org/licenses/by-nc-nd/2.5/ 


\title{
Large Eddy Simulation of a Controlled Diffusion Compressor Cascade
}

\author{
W.A.McMullan · G.J. Page
}

Received: date / Accepted: date

\begin{abstract}
In this research a Controlled Diffusion (CD) compressor cascade stator blade is simulated at a Reynolds number of $\sim 700,000$, based on inflow velocity and chord length, using Large Eddy Simulation (LES). A wide range of flow inlet angles are computed, including conditions near the design angle, and at high negative and positive incidence. At all inlet angles the surface pressure distributions are well-predicted by the LES. Near the design angle the computed suction side boundary layer thickness agrees well with experimental data, whilst the pressure side boundary layer is poorly predicted due to the inability of LES to capture natural boundary layer transition on the present grid. A good estimation of the loss is computed near the design angle, whilst at both high positive and negative incidences the loss is less well predicted owing to discrepancies between the computed and experimental boundary layer thickness. At incidences above the design angle a laminar separation bubble forms near the leading edge of the suction surface, which undergoes a transition to turbulence. Similar behaviour is noted on the pressure surface at negative incidence. At high negative incidence contra-rotating vortex pairs are found to form around the leading edge in

The authors would like to thank Rolls-Royce plc and the UK Technology Strategy Board for the funding this work under the CFMS Core Programme (TP/L3001H). The simulations in this study were performed on HECToR, the UK National Supercomputing Facility, under the UKAAC-2 Framework, EPSRC grant number EP/F005954/1.

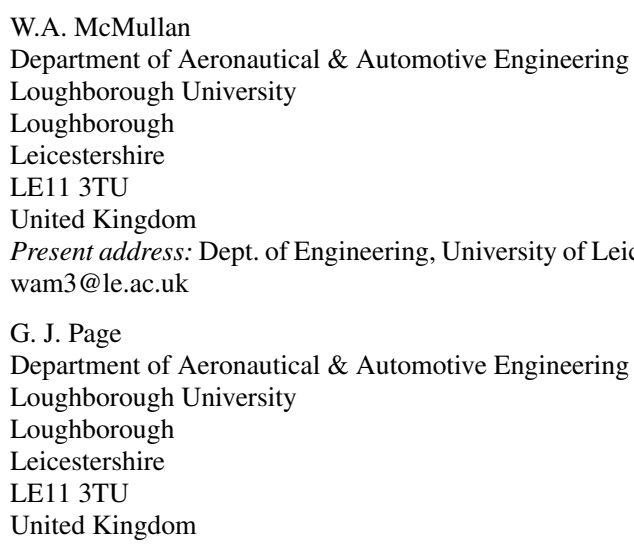


response to an unsteady stagnation line across the span of the blade. Such structures are not apparent in time-averaged statistical data due to their highly-transient nature.

Keywords Large Eddy Simulation · Compressor · Transition · Vortex Structure

\section{Introduction}

In recent years the design of gas turbine compressor components has been complemented by the emergence of Computational Fluid Dynamics (CFD) in the design process. In a design context CFD must be capable of producing accurate flow solutions within a very strict timeframe, and this has limited the CFD techniques used in the design process to ReynoldsAveraged Navier-Stokes (RANS) methods. As the desire for more efficient gas turbines and, in particular, civilian aircraft engines becomes an increasing focus of research, the margins for improvements in efficiency through design advances have become increasingly small. In order to further understand the physical processes which lead to loss in turbomachinery designs a computational method that offers both a high spatial resolution and time-accurate description of the flow is required. Whilst fully-resolved Direct Numerical Simulation (DNS) will remain limited to moderate Reynolds number flows in simple geometries for the foreseeable future [1], [2], advances in modern super-computing power have made both Large Eddy Simulation (LES) and Detached Eddy Simulation (DES) viable options for industrial use.

The majority of published research has focused on simulations of the mid-span of blades in linear cascades. Whilst computationally expensive, DNS has been performed for such turbine cascades at transitional Reynolds numbers [3]. LES of turbine cascades have been performed at conditions that match reference DNS data [4], and it was found that the LES predicted a transition on the suction side of the blade that was delayed by some $10 \%$ when compared to the DNS. This was attributed to the coarse grid used in the simulation and highlights the resolution requirements for wall-bounded simulations. In all of the above simulations the incoming wakes were included in the simulations through the use of precursor calculations [5]. The effect of small-scale turbulence embedded within the wakes was studied by Wissink et al. [6], who concluded that whilst the large-scale motion of the wake triggers the Kelvin-Helmholtz $(\mathrm{K}-\mathrm{H})$ instability on the suction side of the blade, the small scale disturbances embedded into the wake flow seed the transition to turbulence in the shear layer flow.

Relatively few published studies of compressor-type flows have been performed using LES. Pioneering work in the area by You et al. [7] established a computational framework within which Large Eddy Simulations of the loss mechanisms in rotor tip-clearance flows were investigated [8], including the effect of varying tip-gap size [9]. Based on the findings of this work, further efforts were made to improve turbulence modelling of the flow in turbomachinery [10]. The influence of freestream turbulence on transition on turbine blades has been investigated using LES [11], highlighting the need for reasonably posed inlet conditions. Simulations of a linear compressor cascade at a moderate Reynolds number was studied by Zaki et al. [12] - the periodically passing wake caused the suction-side flow to separate and roll-up into discrete $\mathrm{K}-\mathrm{H}$ vortices. On the pressure side the flow underwent a bypass transition due to the passing of the wake.

In a more generic context LES has been extensively used to simulate flow features that occur in turbomachinery using simple geometries. The laminar separation and transition to turbulence of a boundary layer have been simulated both on a flat plate [13] and on a geometry that includes a curved leading edge [14]. In both cases the simulation results compared 
well with experiment and elucidated the transition mechanism in the flow. Wake-induced transition of a flat-plate boundary layer has been simulated numerically [15] with the transition mechanism captured in the simulation comparing very favourably with experiment. A study of the wake produced by two turbulent boundary layers has shown that LES can produce extremely good agreement with experimental data for this flow type [16].

The simulations presented here are performed on a geometry that is restricted to an idealised, highly-loaded compressor blade [17] at a Reynolds number of $\sim 700,000$ based on chord length and inlet velocity $V_{i}$. Many numerical studies on this compressor have been performed but to date the cascade has not been the subject of a systematic study using Large Eddy Simulation. Previous studies of Large Eddy Simulations performed on the cascade has highlighted the computational domain and mesh resolution requirements for the simulation methodology [18], [19]. The purpose of the current study is to identify the relative strengths and weaknesses of the LES method in predicting the flow in an idealised compressor at reasonably high Reynolds number. The simulated flow conditions are across a wide range of flow inlet angles that represent the near-design and off-design points of the blade.

This work uses the Rolls-Royce CFD code Hydra [20] which is an unstructured, mixed element, compressible, density-based Reynolds Averaged Navier-Stokes solver. For the LES implementation, the discretisation has been improved so as to avoid excessive dissipation of resolved eddies, and subgrid scale models incorporated. The important features are summarised below, and further details of the discretisation and testing on simpler LES flow problems can be found in Tristanto et al. [21]. The method has also been validated on multiple impinging jet problems [22] and the complex geometry capability demonstrated by the calculation of a Harrier aircraft in ground-effect [23].

\section{Governing Equations}

Employing Cartesian tensor notation and the conservative variables $\left(\rho, \rho u_{i}, E\right)$, the governing time dependent equations in terms of spatially filtered, Favre-averaged compressible N-S equations can be expressed as

$$
\frac{\partial}{\partial t} \int_{\Gamma} Q d V+\int_{\partial \Gamma} F(Q) \cdot n d S+\int_{\partial \Gamma} G(Q) \cdot n d S=0
$$

where,

$$
Q=\left[\begin{array}{c}
\bar{\rho} \\
\bar{\rho} \widetilde{u} \\
\bar{\rho} \widetilde{v} \\
\bar{\rho} \widetilde{w} \\
\widetilde{E}
\end{array}\right], F(Q) \cdot n=\left[\begin{array}{c}
\bar{\rho} \widetilde{U}_{n} \\
\bar{\rho} \widetilde{U}_{n} \widetilde{u}+\hat{n}_{x} \bar{p} \\
\bar{\rho} \widetilde{U}_{n} \widetilde{v}+\hat{n}_{y} \bar{p} \\
\bar{\rho} \widetilde{U}_{n} \widetilde{w}+\hat{n}_{z} \bar{p} \\
\widetilde{U}_{n}(\widetilde{E}+\bar{p})
\end{array}\right]
$$

and $G(Q)$ contains viscous and conduction flux terms. The finite volume discretisation provides an implicit filter for the large eddies where - denotes unweighted filtered variables and $\sim$ density weighted filtered variables. The spatial filter size is computed at every node from the control volume surrounding the node. The finite volumes are created from the median-dual of the original unstructured mesh which may contain tetrahedra, hexahedra, pyramids and prisms. 


\subsection{Discretisation}

The fluxes through the median dual control volume faces are accumulated by looping over all the edges connecting the nodes. For an edge $i j$ that connects nodes $i$ and $j$, the flux is computed using a second-order accurate scheme of Moinier [24]

$$
F_{i j}=\frac{1}{2}\left[F\left(Q_{i}\right)+F\left(Q_{j}\right)-\text { smoothing }\right],
$$

The smoothing term is defined as [20],

$$
\text { smoothing }=\left|A_{i j}\right| \varepsilon\left(L_{j}^{l p}(Q)-L_{i}^{l p}(Q)\right),
$$

where $L^{l p}$ is the pseudo-Laplacian and

$$
\left|A_{i j}\right|=\partial F / \partial Q
$$

For LES it is essential that the smoothing term should be kept as small as possible so as to avoid unphysical dissipation of the resolved eddies. Careful examination of the splitting and least-squares linear reconstruction of the smoothing terms in test cases [25], [26], showed that there was a lower 'floor' in the effective smoothing coefficient and that equivalent results (for a pipe and free jet) could be obtained with a fixed smoothing coefficient $\varepsilon=0.2$.

Temporal discretisation uses a standard third order accurate, three-stage Runge-Kutta algorithm.

\subsection{Sub Grid Scale Model}

The standard Smagorinsky SGS model [27] defines the subgrid scale viscosity as

$$
\mu_{s g s}=\rho l_{\text {smag }}^{2} \sqrt{2 S_{i j} S_{i j}},
$$

where the Smagorinsky length scale is,

$$
l_{\text {smag }}=C_{s} \Delta
$$

the strain rate is,

$$
S_{i j}=\frac{1}{2}\left(\frac{\partial u_{\mathrm{i}}}{\partial x_{\mathrm{j}}}+\frac{\partial u_{\mathrm{j}}}{\partial x_{\mathrm{i}}}\right),
$$

the filter width $\Delta$ is determined from the cube root of the control volume and $C_{S}$ is a model constant.

A typical hexahedral mesh used for engineering calculations resolves the gradients in the boundary layer by decreasing spacing normal to the wall. This results in moderate to high aspect ratios close to the wall. The consequence is that the filter width stays relatively large and excessively high values of $\mu_{s g s}$ are found close to the wall. In order to reduce the eddy viscosity near the wall, a damping algorithm is implemented into the code. In the inner region of the Baldwin-Lomax or Cebeci-Smith mixing length RANS model, the turbulent viscosity takes a similar form to the sub-grid scale viscosity of the Smagorinsky model

$$
\left(\mu_{t}\right)_{i}=\rho l_{b l}^{2}|\omega|
$$


where the mixing length is in this case

$$
l_{b l}=\kappa y\left[1-\exp \left(\frac{y^{+}}{A^{+}}\right)\right]
$$

Since the Smagorinsky SGS model only accounts for the modelled part of the total stress, this should be smaller than the Reynolds stress that would be predicted by a mixing length model. Hence, we have a way of limiting the spurious excessive length scale in the Smagorinsky model,

$$
l_{\text {smag }}=\min \left(C_{s} \triangle, \kappa y\right)
$$

This can be interpreted as performing a similar operation to van Driest damping which reduces the length scale to zero near to the wall. Although the limiter employs a RANStype model to compute an upper bound on the length scale near the wall, the subgrid-scale viscosity is calculated at every time step, and the simulation remains as a pure LES. In testing on grids used here, this length scale restriction is typically activated for a layer of 2-5 elements from the wall with the outer element having a $y^{+}$less than seven.

The unstructured nature of the solver requires an efficient partitioning strategy and careful handling of the message passing to achieve good efficiency on large numbers of processors. The unstructured solver uses the OPLUS library [28] with message passing subsequently implemented in MPI. The partitioning is carried out in parallel using the ParMetis library. More information is provided by Hills [29] on how the parallel implementation has been tuned for large scale problems and near linear speed-up is demonstrated up to 1024 cores on an IBM Power5 system. Calculations presented here have been run on a Cray XT-4 system using 1024 processor cores.

\section{Reference Experiment and Computational Setup}

The numerical method is applied to a Controlled Diffusion (CD) compressor cascade housed at the Naval Postgraduate School, Monterey, California. This cascade has been the subject of an extensive experimental testing programme over a wide range of the flow inlet angle, $\beta$, and over a range of Reynolds numbers $R e=400,000-770,000$ based on the freestream velocity and the chord length, $c=0.1273 \mathrm{~m}$. In this study, a cross-section of the experiments on the rig [30], [31], [32] are simulated numerically. The cases investigated are outlined in Table 1 . Cases 1 and 2, where the inflow angle $\beta=24.49^{\circ}$ and $28^{\circ}$ respectively is lower than the design angle are referred to as negative incidence simulations. Cases 6-9, where the inflow angle is above the design point are referred to as positive incidence simulations.

The computational domain and an outline of the mesh used in the study is shown in Figure 1, with only every other grid line shown for clarity. The global co-ordinate system is Cartesian in nature, such that the $x-, y-$, and $z-$ co-ordinates are global and have components of velocity associated with them that are $u, v$, and $w$ respectively. The flow inlet angle, $\beta$, is defined as zero along the positive $\mathrm{x}$-direction and increases in a clockwise sense. The domain extends $0.25 \mathrm{c}$ upstream and $0.75 \mathrm{c}$ downstream of the leading and trailing edges of the blade respectively. The spanwise domain has an extent of $0.2 c$, with 400 nodes distributed uniformly across the span. The domain is derived from a streamtube representation of the blade and contains a contraction of the spanwise domain with axial distance such that the Axial Velocity Density Ratio (AVDR) has a value of 1.025 , matching that of the $\beta=$ $40^{\circ}$ experimental configuration. In the experimental configuration the AVDR was found to remain roughly constant in Cases 7-9, as reported by Elazar [33]. 
Table 1 Flow Simulation Parameters

\begin{tabular}{cccc}
\hline Case & $\beta\left(^{\circ}\right)$ & $M_{1}$ & $\mathrm{Re}$ \\
\hline 1 & 24.49 & 0.2206 & 690,000 \\
2 & 28.00 & 0.2151 & 648,000 \\
3 & 32.95 & 0.2079 & 630,000 \\
4 & 37.07 & 0.2205 & 683,000 \\
5 & 38.91 & 0.2164 & 676,000 \\
6 & 42.90 & 0.2221 & 686,000 \\
7 & 45.96 & 0.2224 & 690,000 \\
8 & 40.00 & 0.2500 & 740,000 \\
9 & 43.40 & 0.2724 & 774,000 \\
\hline
\end{tabular}

The number of axial nodes is set to 370 on each surface of the blade, with nodes clustered towards the leading and trailing edges. The grid normal to the blade surface has a first-cell height that gives a $y^{+} \approx 4$ at the leading edge, and $y^{+} \approx 2.2$ at the trailing edge. The minimum $y^{+} \approx 1.6$ at approximately $60 \%$ chord on the pressure-side surface. The spanwise spacing $z^{+} \approx 16$, and the streamwise spacing varies between $x^{+} \approx 15$ at the leading and trailing edges to $x^{+} \approx 50$ at mid-chord. There are a total of 39.6 million nodes in the computational domain. The blade surface is a viscous wall and has a no-slip boundary condition applied to it. The lateral boundaries are inviscid endwalls as the contraction due to the non-unity AVDR prevents the use of periodic boundaries in this direction. The upper and lower boundaries are periodic surfaces. For each flow inlet angle one-dimensional total pressure and temperature profiles are applied at the inlet plane. Pseudo-random fluctuations with an intensity of $1.4 \%$ are applied to the inlet plane at each time step in order to model the freestream turbulence reported in the experiment. Fluctuations are imposed on each of the three velocity components. A static pressure boundary condition is used at the outflow of the computational domain.

The Smagorinsky constant is set to $C_{s}=0.1$ for all runs. The time step for the computations is $5.8 \times 10^{-5} c / V_{\text {ref }}$, where $V_{\text {ref }}$ is the inlet velocity for Case 8 . The time step is chosen such that four time steps are required for a parcel of fluid to pass through the smallest characteristic length-scale in the grid. In order to avoid the high computational cost involved in propagating starting transient flow features through the domain the initial LES flow field is obtained from a suitably-converged RANS solution. Statistical samples were obtained every ten time steps over a period of 300,000 time step increments, and the simulation run-time was $\sim 48$ hours on 256 AMD 2.3GHz Opteron quad-core CPUs, with a total of 1024 cores used.

In the following sections all statistical velocity data are normalised by the inlet velocity of the simulation, $V_{i n}$, following the normalisation procedure used in the experiment. The boundary layer profiles are presented in a normal co-ordinate system, $r$, that is local to the blade surface at each measuring station, with the velocity tangential to the blade surface denoted $u_{t}$. All instantaneous visualisation data are presented in the global co-ordinate system, as a function of tangential chord length $c_{t}$. The surface pressure coefficient, $C_{p}$, is defined as

$$
C_{p}=\frac{p-p_{0}}{\frac{1}{2} \rho V_{i n}^{2}}
$$

where $p_{0}$ is the static pressure at the inlet boundary. Case 8, with an inflow angle of $\beta=40^{\circ}$, has been selected for detailed comparison with the reference data as it is an inflow 
angle near to on-design conditions that has a large amount of experimental data available [31]. Statistical data from the other cases will be shown where appropriate.

4 Case 8, $\beta=40^{\circ}$

The surface pressure distribution obtained from the LES is shown in Figure 2, along with the experimental data. The agreement between simulation and experiment is very good, with the LES predicting a small laminar separation bubble near the leading edge on the suction side. Upon reattachment the suction side boundary layer is turbulent in nature and remains attached over the remainder of the blade. As the mesh resolution near the blade surface matches a previous study into the cascade [18], the fidelity of the grid avoids any unexpected separation that occurs in the suction side boundary layer on poorly-resolved grids at this flow inlet angle. Inspection of instantaneous flow data highlights the presence of the laminar separation bubble near the leading edge on the suction side of the blade. This behaviour of the suction side boundary layer was also noted in the experiment and will be discussed further in Section 6.

Boundary layer profiles recorded at mid-span from three locations on the suction side of the blade are shown in Figure 3. Near the leading edge the LES shows very good agreement with the experimental data, whilst towards the trailing edge the profile showing some disparity with experiment, with the gradient of tangential velocity near the wall being substantially over-predicted. Similar boundary layer velocity distributions have been found in other numerical studies of the cascade [18], [33], [34], [35] although the reasons for these discrepancies are not fully understood. The boundary layer profiles can be used to obtain the edge velocity of the flow, defined as the maximum velocity in the boundary tangential to the blade surface. On the suction side this value is easily determined through the familiar $\delta=0.99 U_{\infty}$ estimation, as can be seen in the boundary layer profiles of Figure 3. This technique matches that used in the experimental investigation of this case [31]. However on the pressure side the irrotational curved stream introduces some difficulty in determining the boundary layer thickness, hence the approach used by Chen et al. [34] is employed, such that

$$
\delta=\left.1.936 r\right|_{(r|d u / d r|)_{\max }}
$$

with the edge velocity computed accordingly. For Case 8 the LES prediction of the edge velocity is in excellent agreement with the experimental data, as shown in Figure 4.

Statistical properties of the boundary layers on the blade surfaces at mid-span are given in Figure 5. The boundary layer thickness on the suction side is predicted well by the LES in Figure 5a, with the maximum thickness of the boundary layer at the trailing edge being $\delta=0.042 c$. This value is one-fifth of the spanwise extent of the computational domain and indicates that the growth of structures within the boundary layer is not inhibited by the spanwise boundaries [19]. On the pressure side the LES prediction of the boundary layer thickness is quite poor, being significantly smaller than that reported in the experiment. The simulation under-predicts the displacement thickness of the suction side boundary layer, a feature that is common throughout both RANS and coupled boundary-layer numerical studies that have simulated this CD blade [33], [34], [35]. On the pressure side the comparison between simulation and experiment is reasonably good. The reason for the poor prediction of the boundary layer thickness on the pressure side is apparent in Figure 5c, as the shape factor distribution of the experimental data shows that the boundary layer underwent a natural transition to turbulence aft of mid-chord. The LES is unable to resolve the instability 
waves required to precipitate a natural transition to turbulence, and as such the shape factor of the flow suggests laminar boundary layer behaviour over the majority of the pressure-side surface. The value of the shape factor drops to $H \approx 1.6$ at the trailing edge, suggesting that the boundary layer is at least transitional at this location. Analysis of the mean axial velocity near the pressure surface shows that axially-orientated streaks appear in the boundary layer, as demonstrated in Figure 6. The streaks that are apparent in the pressure side boundary layer appear to be Görtler vortices that arise due to the concavity of the pressure surface, but do not precipitate a transition to turbulence in the boundary layer flow. These observations demonstrate the difficulty in using LES to simulate a high Reynolds number flow undergoing natural transition, as producing a mesh that resolves the Tollmien-Schlichting waves that produce the transition would lead to a simulation that is closer to Direct Numerical Simulation than LES.

\section{Off-Design Simulations}

Representative surface pressure distributions from Cases 1, 6 and 9 are shown in Figure 7. The overall agreement between the LES and the experiment is very good over the range of flow inlet angles simulated in this study. It should be noted that some of the experimental data points between $0.8 c-0.9 c$ on the suction surface of the blade appear to be unreliable. The spike in the data in the data of Koyuncu [30] in Figures 7a-b in this region is not replicated in the later studies of the compressor by both Elazar (Figure 2) and Shreeve et al. (Figure 7c).

Boundary layer statistical properties at mid-span from Case 7 are shown in Figure 8, where $\beta=45.96^{\circ}$. At high positive incidence, the trends of the pressure side boundary layer data are similar to that of Case 8 , with the simulated boundary layer remaining laminar across the entire extent of the blade. On the suction side, however, the boundary layer thickness is not as well-predicted by the LES at this incidence angle. This under-prediction of the boundary layer thickness at $\beta=45.96^{\circ}$ is common in coupled-boundary layer studies of the cascade [33], [35]. The trends in the displacement thickness and shape factor of the suction side boundary layer at this incidence angle are also similar to Case 8, with the displacement thickness being significantly under-predicted.

Of particular interest to the design process is the estimation of the total pressure loss coefficient, $\omega$, as a function of flow inlet angle. This coefficient is defined as

$$
\omega=\frac{P_{T 0}-P_{T 2}}{P_{D 1}}
$$

where $P_{T 0}$ and $P_{T 2}$ are the averaged total pressures at the inlet and exit planes respectively, and $P_{D 1}$ is the averaged dynamic pressure at the inlet. In all simulations $P_{T 2}$ is obtained at a plane $0.01 c$ upstream of the exit boundary, in order to avoid any potential contamination from the exit boundary condition. The variation of this coefficient with distance across the span of the domain is shown in Figure 9 for Case 9. Across the mid-span of the blade a relatively uniform loss is obtained from the calculation, but towards the endwalls the computed loss increases rapidly. This is due to the interaction between the vortical structures captured by the LES and the inviscid wall - the structures impinge on the inviscid wall and are displaced along the surface, resulting in an increase in the boundary layer thickness and hence the loss. To produce a reliable estimation of the loss coefficient from the LES cases, $15 \%$ of the span near each inviscid boundary is neglected in the averaging procedure. 
The loss coefficient for each case is shown in Figure 10, with a comparison to the linear model RANS data of Chen et al. [34]. Whilst the overall trend of the loss predicted by the LES is in reasonable agreement with the experimental data, some discrepancies are evident. The under-prediction of the boundary layer thickness in Case 7 by approximately $40 \%$ (Figure 8) results in the lower loss estimate from the LES. In Case 9 the computed suction side boundary layer thickness is also too small when compared to experimental data. Similarly the boundary layers predicted in Cases 1-4 are too thick when compared to experimental data, hence the loss obtained from these simulations is slightly too high. Some of the discrepancies in the computed loss may arise from the constant value of the AVDR used in the simulations, as the experimental data showed a small variation in this quantity over the wide range of flow angles studied in this research [36]. As shown by the comparative data of Chen et al., RANS predictions of the loss of the compressor cascade have shown similar trends in the variation of loss with flow inlet angle and emphasises the difficulty involved in accurately predicting the loss through both steady-state Computational Fluid Dynamics, and time-dependent numerical simulation. As the LES captures the separationinduced transition in the flow, however, the obtained loss loop does show some improvement over traditional RANS modelling methods in the prediction of this quantity.

\section{Instantaneous Flow Structure}

The time-dependent nature of LES allows insights into the development of blade surface boundary layers to be obtained. Sequences of instantaneous flow outputs and the mean flowfield from each case are analysed in order to elucidate both the mechanism of transition to turbulence in the flow, and any structure that may be present in the turbulent flow field. Near the extremes of negative and positive flow angles the flow will undergo a transition on the pressure and suction surfaces respectively, and as such the discussion of the analysis will be split between those regions.

\subsection{Negative Incidence}

In Cases 1-3 the mean flow field data reveals the presence of laminar separation near the leading edge on the pressure-surface of the blade, and the structure of the flow in these cases is investigated further. The instantaneous global axial velocity, $u$, at a distance of $r / c=$ $8.9 \times 10^{-4}$ from the blade surface in Case 2 is shown in Figure 11. At this particular instant in time the flow separates near the leading edge of the pressure surface (Figure 11a) and reattaches at approximately $x / c_{t}=0.08$. Upon reattachment axially-orientated low-velocity streaks form in the boundary layer and persist to the trailing edge of the blade. This pattern of flow development is very similar to that observed in DNS of boundary layers undergoing laminar separation and turbulent reattachment [37]. On the suction surface, shown in Figure $11 \mathrm{~b}$ the flow is laminar up to $x / c_{t}=0.53$, at which point the flow undergoes a transition to turbulence, prompting the appearance of streaks in the boundary layer flow. The flow structure near the leading edge on the pressure surface is visualised in Figure 12, through the use of the $Q$-criterion [38]. The Q-criterion is given by the expression $Q=-0.5 u_{i j} u_{j i}$, with the threshold of $Q$ chosen to visualise the features near the leading edge of the blade. The shear layer that develops from the separating flow is inviscidly unstable and rapidly forms Kelvin-Helmholtz vortices. These vortices initially display a high level of spanwise 
coherence, but are quickly broken down as they proceed downstream. As the flow reattaches to the blade surface, hairpin vortices are evident in the boundary layer flow.

The flow inside the separation bubble of Case 2 is shown in Figure 13, with only half of the span of the blade shown for clarity. As the oncoming stream of fluid separates from the blade the flow within the bubble at $x / c_{t}=0.0164$ it is reasonably uniform, with no significant three-dimensionality indicated by the vectors of $v$ and $w$ velocity components. At $x / c_{t}=0.0328$ the flow in the separation bubble displays evidence of three-dimensional motion, with these fluctuations perturbing the spanwise coherence of the separating shear layer. Further downstream the velocity vectors display evidence of structure within the separation bubble as the shear layer structure loses its coherence. Regions of higher-velocity fluid are transported towards the blade surface by these structures, and regions of reverse flow are similarly transported away from the wall (Figure 13d). These results are in good agreement with previous numerical and experimental studies of the separated flow on a flat-plate [14], [37], [39]. Figure 14 shows the approximate location where the flow reattaches at this given instant in time. It is also evident that the boundary layer displays a high level of intermittency across the span of the blade. This pattern is repeated along the remainder of the extent of the pressure surface.

In Case 1 , which has the highest negative incidence angle, $\beta=24.49^{\circ}$, the evolution of the flow near to the leading edge of the blade is much different to that of the other negative incidence simulations. A plot of the instantaneous axial velocity, $u$, at a distance of $r / c=8.9 \times 10^{-4}$ from the blade surface is shown in Figure 15. Whilst the flow on the pressure surface in Figure 15a is qualitatively similar to that in Case 2, the suction-side flow in Case 1(Figure 15b) displays evidence of streaks in the boundary layer at the leading edge. These streaks suggest that streamwise vorticity is present at the leading edge of the flow. In Figure 16, the $Q$ - iso-surface reveals that axially-orientated vortices are wrapped around the leading edge of the blade. The K-H vortices that develop from the free shear are severely distorted by the presence of these vortex structures and quickly break down into three-dimensional structures. In addition the streaks that wrap around the leading edge do not remain in a fixed position with time; instead they are observed to shift along the span of the blade. The presence of these streaks can be traced to the response of the fluid to the stagnation line across the leading edge of the blade. The static pressure distribution along the leading of the blade in Case 1 at two arbitrary instants in time is shown in Figure 17a, along with the mean data. The instantaneous data shows a high degree of temporal variability in the surface pressure at the leading edge, indicating that the stagnation line across the blade is unstable, and subsequently the stagnation line does not occur in a fixed position across the span of the blade on an instantaneous basis. The mean flow surface pressure data is, by contrast, almost constant across the blade span, as would be expected for suitably-converged time-averaged samples. When the mean velocity streamlines are plotted with a visualisation of the flow structure in Figure 18 it is evident that the structures form around the mean stagnation line of the blade. The temporal variability in the stagnation line forces a response in the fluid upstream of the blade, resulting in the formation of vortices that stretch over the leading edge and influence the blade surface boundary layers.

Data from the stagnation line of Case 2 is presented in Figure 17b, which shows that there is little variation between the instantaneous pressure distribution in this region and the time-averaged mean data. Similar pressure distributions are found at all recorded time instants in Case 2, and flow visualisations such as Figure 12 confirm that no vortex structures are present around the leading edge of the blade at this flow angle. Case 1 is the only simulation in the current study that displays evidence of streaks which wrap around the leading edge. 
The analysis of the mean and instantaneous flow data obtained from Case 1 highlights an important consequence of statistical averaging and the elucidation of coherent vortex structures in fluid flow; from the mean statistical data presented in this research it is practically impossible to conclude that the flow structure in Case 1 is substantially different to any of the other cases simulated - it is only through flow visualisation and the analysis of fully-unsteady data that such structures have been educed. The highly transient nature of the contra-rotating vortex pairs which wrap themselves around the leading edge would also prevent oil-flow or china-clay visualisation techniques from producing any evidence of such structures around the leading edge. This is in contrast to the axially-orientated structures in the boundary layer of yawed bodies which have a regular pattern of development [40].

\subsection{Positive Incidence}

Representative instantaneous axial velocity distributions of the flow at a distance of $r / c=$ $8.9 \times 10^{-4}$ from the suction surface in the positive incidence cases are shown from Case 9 in Figure 19. A laminar separation bubble is present near the leading edge of the blade, shown as the light patch in Figure 19. The laminar separation bubble is present for all flow angles above the design angle, and the length of the bubble increases with increasing flow angle. The instantaneous position of reattachment is also observed to temporally shift along the blade surface, indicating a high degree of variability with the separation bubble. The lowspeed streaks near to the blade surface are also observed on the suction surface at positive incidence, implying the presence of contra-rotating vortex pairs on a convex surface. Similar structures have been found on turbine blades, and a recent summary of the evidence for their existence has been presented by Gostelow et al. [41].

The vortex structure in the region of the separation bubble is highlighted using the $Q$-criterion in Figure 20. The development of the suction-side separation bubble for all positive incidence inflow angles is very similar to that found on the pressure surface in Case 2 described above - initially coherent K-H vortices are shed by the shear layer, which are then rapidly broken down into three-dimensional hairpin vortices as the flow reattaches to the blade surface. At all positive incidence angles there is no evidence of the formation of contra-rotating vortex pairs around the leading edge, as are observed in Case 1.

As was discussed in Section 4 the flow on the pressure side remains laminar over the entire chord at positive incidence. Görtler-type counter-rotating vortices are noted near the trailing edge of the blade, but these do not cause a transition to turbulence in the boundary layer at any positive incidence angle.

\section{Conclusions}

A systematic study of a Controlled Diffusion compressor cascade has been performed using Large Eddy Simulation, over a wide range of flow inlet angles at a Reynolds numbers of Re $\sim 700,000$, based on chord length. The computed surface pressure distributions for all cases agree well with experimental data, particularly at both highly positive and negative incidence angles, far from the design point of the blade. The boundary layer-thickness, particularly on the suction-surface, is well-predicted by LES near the design angle, but less well-predicted at off-design angles. The LES fails to resolve the natural boundary layer transition on the pressure surface at positive incidence, a result that may be expected for the grid resolution used in this study. Near the design angle the loss computed by the LES is in reasonable 
agreement with experimental data, but at off-design angles the inadequate boundary layer thickness prediction results in discrepancies in the calculated loss.

Inspection of the vortex structure in the flow reveals that a laminar separation bubble forms near the leading edge of the pressure- and suction-side when the flow is at negative and positive incidence respectively. In the majority of cases the shear layer that forms above the laminar separation bubble sheds spanwise-coherent Kelvin-Helmholtz vortices which are rapidly broken down into three-dimensional structures. Hairpin vortices are observed to form downstream of the reattachment point, and low-speed streaks are evident in the boundary layer from the reattachment point up to the trailing edge of the blade. At extreme negative incidence, axially-orientated vortex structures are found to form around the leading edge of the blade, in response to an unsteady stagnation line across the blade span. The location of the formation of the vortex pairs is highly transient and the mean statistical data of the flow around the leading edge offers no direct evidence of their existence, in spite of their overall influence on the development of the flow. The lack of evidence of these structures in mean statistical data indicates that time-dependent flow visualisation techniques will be required to educe such vortex pairs in experimental researches.

Acknowledgements The permission of Rolls-Royce and CFMS to publish this work is gratefully acknowledged. The authors would like to thank Rolls-Royce plc and the UK Technology Strategy Board for the funding this work under the CFMS Core Programme (TP/L3001H). The simulations in this study were performed on HECToR, the UK National Supercomputing Facility, under the UKAAC-2 Framework, EPSRC grant number EP/F005954/1. One author expresses his gratitude to Professor J. Paul Gostelow for many insightful discussions during the analysis of the data presented here. Thanks are also expressed to Mike Howard of Rolls-Royce plc for assistance in interpretation of the experimental measurements.

\section{References}

1. Wu, X., Moin P., 2000. A direct numerical simulation study on the mean velocity characteristics in turbulent pipe flow. J. Fluid Mech, 50, 27-61.

2. Wang, Y., Tanahashi, M., Miyauchi, T., 2007. Coherent fine scale eddies in turbulence transition of spatially-developing mixing layer. Int. J. Heat Fluid Flow, 28, 1280-1290.

3. Wissink, J.G., Rodi, W., 2006. Direct numerical simulation of flow and heat transfer in a turbine cascade with incoming wakes. J. Fluid Mech., 569, 209-247.

4. Michelassi, V., Wissink, J.G., Frohlich, J., Rodi, W., 2003. Large-Eddy Simulation of Flow Around LowPressure Turbine Blade with Incoming Wakes. AIAA J., 41, 2143-2156.

5. Wu, X. and Durbin, P.A., Evidence of longitudinal vortices evolved from distorted wakes in a turbine passage. J. Fluid Mech., 446, 199-228.

6. Wissink, J.G., Rodi, W., Hodson, H.P., 2006. The influence of disturbances carried by periodically incoming wakes on the separating flow around a turbine blade. Int. J. Heat Fluid Flow, 27, 721-729

7. You, D., Mittal, R., Wang, M., and Moin, P., 2004. Computational Methodology for Large-Eddy Simulation of Tip-Clearance Flows. AIAA J., 42, 271-279.

8. You, D., Mittal, R., Moin, P., and Wang, M., 2004. Effects of tip-gap size on the tip-leakage flow in a turbomachinery cascade. Phys. Fluids, 18, 105102.1-105102.14.

9. You, D., Wang, M., Moin, P., and Mittal, R., 2007. Large-eddy simulation analysis of mechanisms for viscous losses in a turbomachinery tip-clearance flow. J. Fluid Mech., 586, 177-204.

10. Borello, D., Hankalic, K., and Rispoli, F., 2007. Computation of tip-leakage flow in a linear compressor cascade with a second-moment turbulence closure. Int. J. Heat Fluid Flow, 28, 587-601.

11. Matsuura, K., and Kato, C., 2007. Large Eddy Simulation of Compressible Transitional Cascade Flows. J. Fluid Sci. Tech., 2, 558-569.

12. Zaki, T.A., Wissink, J.G., Durbin, P.A., Rodi, W., 2009. Direct Computations of Boundary Layers Distorted by Migrating Wakes in a Linear Compressor Cascade. Flow, Turb. Combust., DOI 10.1007/s10494-009-9216-0

13. Roberts, S.K., and Yaras, M.I., 2007. Large-Eddy Simulation of Transition in a Separation Bubble. J. Fluids Eng., 128, 232-238. 
14. Yang, Z., and Voke, P.R., 2001. Large-eddy simulation of boundary-layer separation and transition at a change of surface curvature. J. Fluid Mech., 439, 305-333.

15. Wu, X., Jacobs, R.G., Hunt, J.C.R., and Durbin, P.A., 1999. Simulation of boundary layer transition induced by periodically passing wakes. J. Fluid Mech., 398, 109-153.

16. Gao, S., Voke, P.R., and Gough, T., 1997. Turbulent simulation of a at plate boundary layer and near wake. Direct and Large Eddy Simulation II, 115-124.

17. Sanger, N.L., 1983. The use of optimization techniques to design controlled-diffusion compressor blading. J. Eng. Power, 105, 256.

18. McMullan, W.A., Page, G.J., 2009, Large Eddy Simulation of a Controlled-Diffusion Cascade Blade at Varying Flow Inlet Angles. ASME GT2009-59668, ASME Turbo Expo 2009, Orlando. FL.

19. McMullan, W.A., Page, G.J., 2010, Loss Coefficient Estimation in a Controlled Diffusion Cascade using Large Eddy Simulation. AIAA 2010-315, 49th AIAA Aerospace Science Meeting, Orlando. FL.

20. Crumpton, P.I., Moinier, P., and Giles, M.G., 1997. An Unstructured Algorithm for High Reynolds Number Flows on Highly Stretched Grids. Tenth International Conference on Numerical Methods for Laminar and Turbulent Flow.

21. Tristanto, I.H., Li, Q., Page, G.J., and McGuirk, J.J., 2006. On the Effect of Convective Flux Formation for LES of Compressible Flows using Hybrid Unstructured Meshes. AIAA Paper 2006-54940, 36TH AIAA Fluid Dynamics Conference, San Francisco, California, June 5-8, 2006.

22. Li, Q., Page, G.J., McGuirk, J.J., 2007. Large-eddy simulation of twin impinging jets in crossflow. Aero. J.l, 111, 195-205.

23. Page, G.J., and McGuirk, J.J., 2009. Large Eddy Simulation of a Complete Harrier Aircraft in Ground Effect/ Areo. J., 113, 99-106.

24. Moinier, P., 1998. Algorithm Developments for an Unstructured Viscous Flow Solver. PhD Thesis, University of Oxford.

25. Ciardi, M., Sagaut, P., Klein, M. and Dawes, W.N., 2005. A dynamic finite volume scheme for large-eddy simulation on unstructured grids. J. Comp. Phys., 210, 632-655.

26. Ducros, F., Ferrand, V., Nicoud, F., Weber, C., Darracq, D., Gacheriu, C., and Poinsot, T., 1999. LargeEddy Simulation of the Shock/Turbulence Interaction, J. Comp. Phys., 152, 517-549.

27. Smagorinsky, J., 1963. General circulation experiments with the primitive equations. Mon. Weath. Rev., 91, 99-164.

28. Burgess, D.A., Crumpton, P.I. and Giles, M.B., 1994. A Parallel Framework for Unstructured Grid Solvers. Programming Environments for Massively Parallel Distributed Systems, 97 -106.

29. Hills, N., 2007. Achieving High Parallel Performance for an Unstructured Unsteady Turbomachinery Code. Aero. J., 111, 185-193.

30. Koyuncu, Y. 1984. Report of tests of a compressor configuration of CD blading. MS Thesis, Naval Postgraduate School, Monterey, CA.

31. Elazar, Y., Shreeve, R.P., 1990. Viscous flow in a controlled diffusion compressor cascade with increasing incidence. J. Turbomach., 112, 256-265.

32. Shreeve. R.P., Elazar, Y., Dreon, J.W., Baydar, A., 1991. Wake measurements and loss evaluation in a controlled diffusion compressor cascade. J. Turbomach., 113, 591-599.

33. Elazar, Y., 1988. A Mapping of the Viscous Flow Behavior in a Controlled Diffusion Compressor Cascade Using Laser Doppler Velocimetry and Preliminary Evaluation of Codes for the Prediction of Stall, Ph. D. Thesis, Naval Postgraduate School, Monterey, CA.

34. Chen, W.L., Lien, D.S., Leschziner, M.A., 1998. Computational prediction of flow around highly loaded compressor- cascade blades with non-linear eddy-viscosity models. Int. J. Heat Fluid Flow, 19, 307-319.

35. Ho, W.K., Walker, G.J., Stow, P., 1990. Boundary Layer and Navier-Stokes Analysis of a NASA Controlled-Diffusion Compressor Blade. ASME Paper No. 90-GT-236.

36. Sanger, N.L., Shreeve, R.P., 1986. Comparison of calculated and experimental cascade performance for controlled-diffusion compressor stator blading. J. Turbomachinery, 108, 42-50.

37. Alam, M., Sandham, N., 2000. Direct numerical simulation of short laminar separation bubbles with turbulent reattachment. J. Fluid Mech., 403, 223-250.

38. Hunt, J.C.R., Wray, A., Moin, P. 1988. Eddies, stream, and convergence zones in turbulent flows. Centre for Turbulence Research Report CTR-S88.

39. Kiya, M., Sasaki, K., 1983. Structure of a turbulent separation bubble. J. Fluid Mech., 137, 83-113.

40. Poll, D., 1985. Some observations of the transition process on the windward face of a long yawed cylinder. J. Fluid Mech., 150, 329-356.

41. Gostelow, J.P., Mahallati, A., Carscallen, W.E., Rona, A., 2010. Organised streamwise vorticity on convex surfaces with particular reference to turbine blades. AIAA 2010-904, 49th AIAA Aerospace Science Meeting, Orlando, FL. 


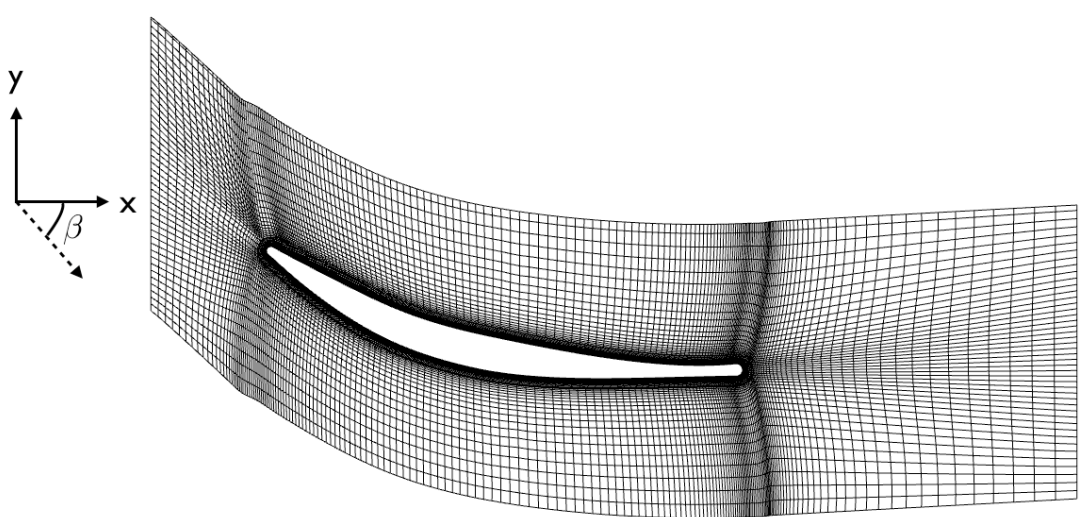

Fig. $1 x-y$ plane through computational domain used in the simulations. Every second grid line shown for clarity. 


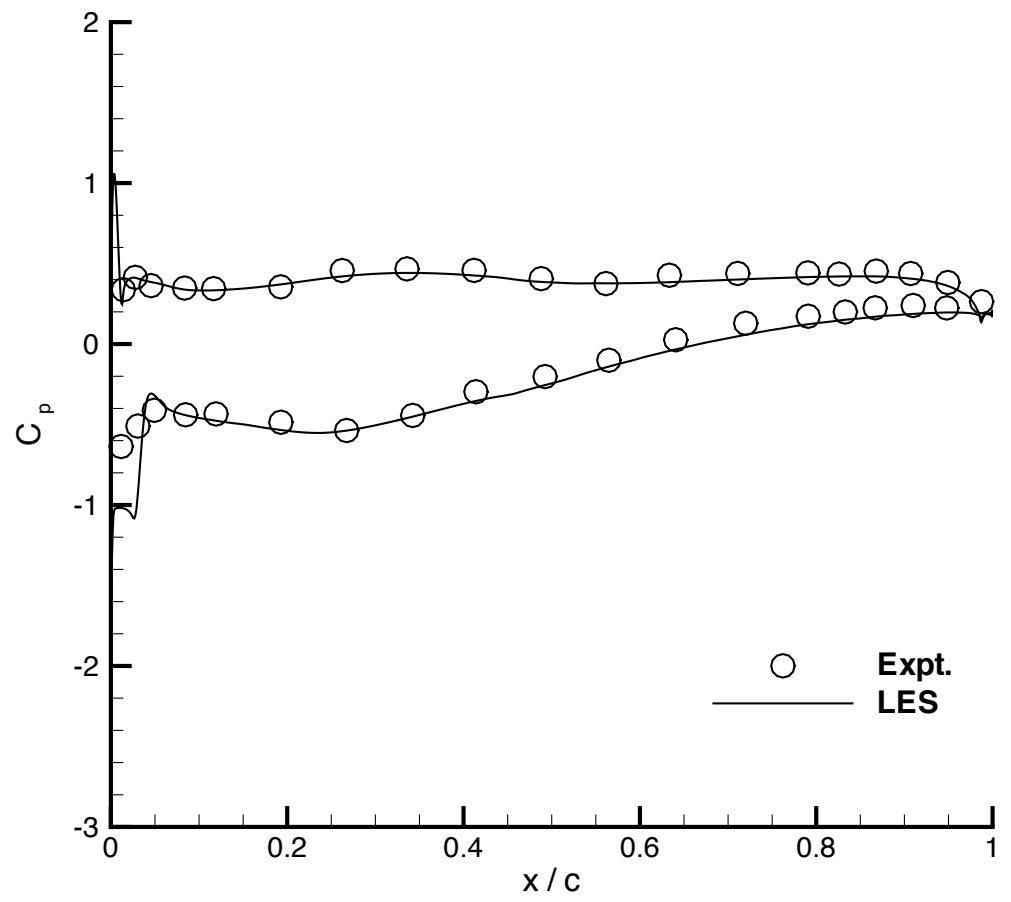

Fig. 2 Case 8 surface pressure distribution, $\beta=40^{\circ}$. Experimental data produced by Elazar and Shreeve [31]. 
16

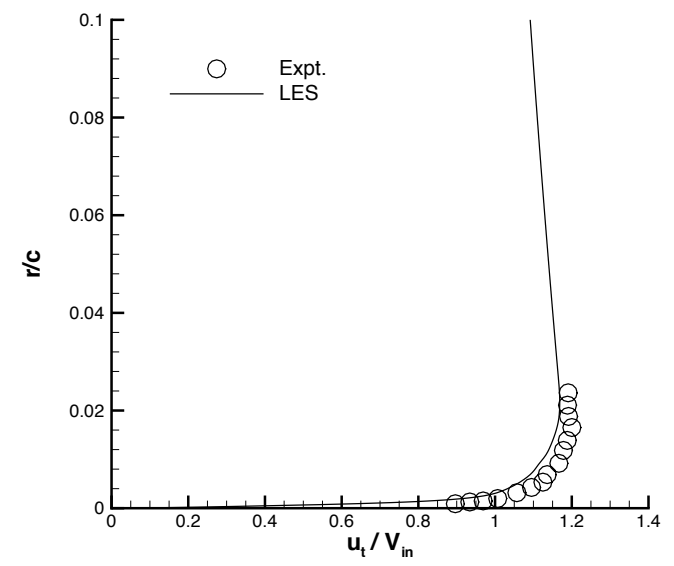

(a) $x / c=0.156$

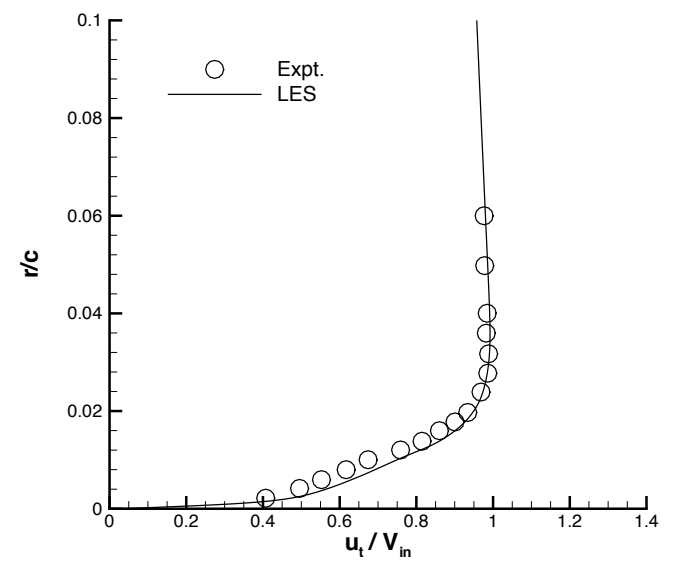

(b) $x / c=0.629$

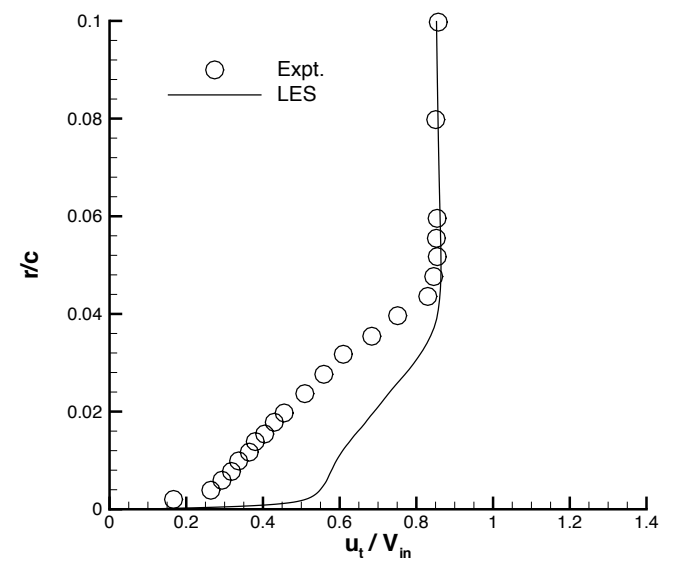

(c) $x / c=1.0$

Fig. 3 Suction side axial velocity profiles for Case 8 . (a) $x / c=0.156$, (b) $x / c=0.629$, (c) $x / c=1.0$. 


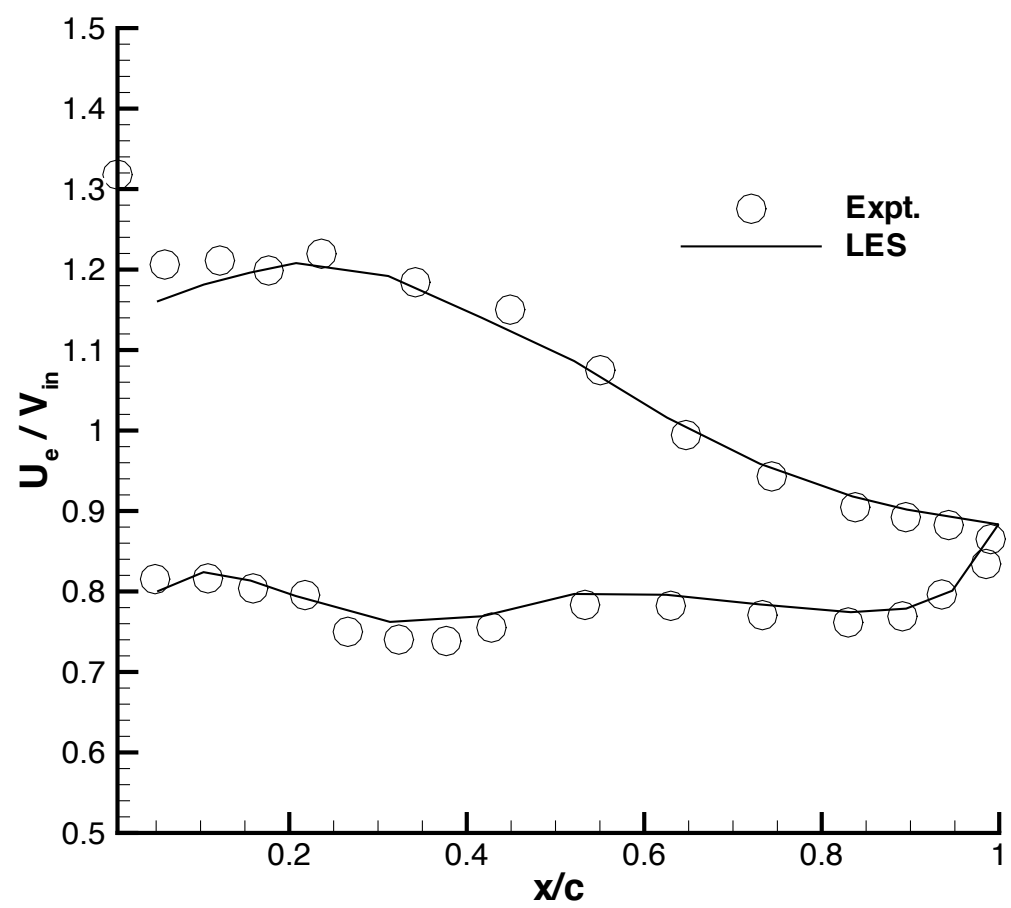

Fig. 4 Edge velocity distribution for Case 8 . 


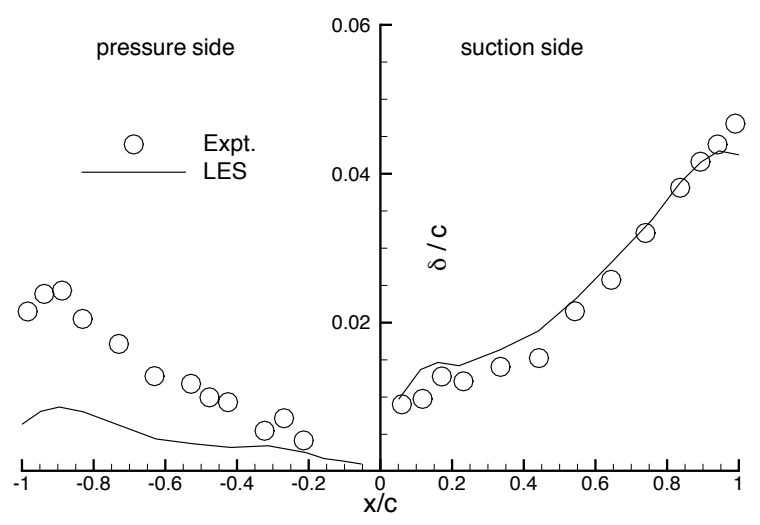

(a) Boundary layer thickness

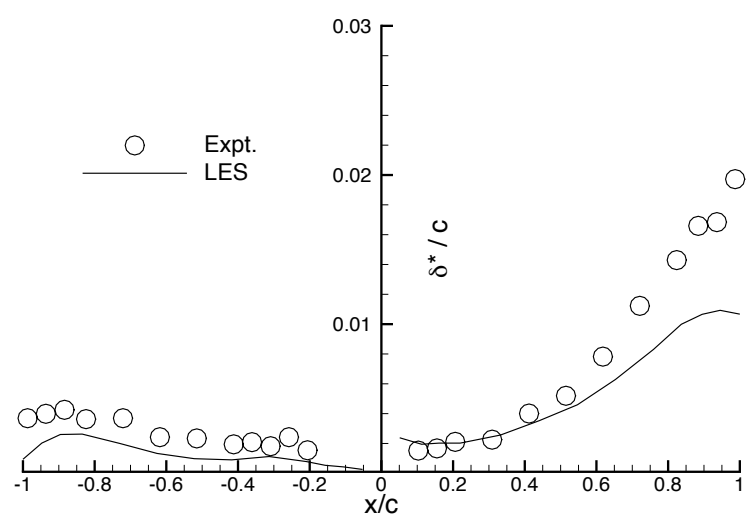

(b) Displacement thickness

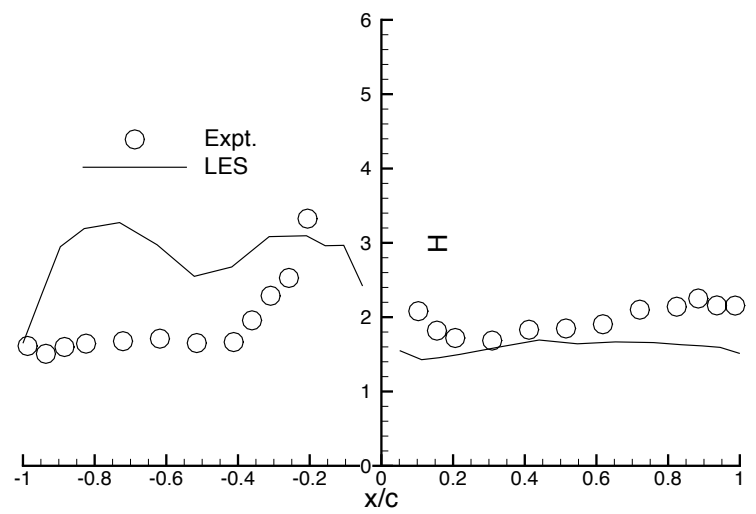

(c) Shape factor

Fig. 5 Boundary layer integral properties for Case 8. (a) Boundary layer thickness, (b) Displacement thickness, (c) Shape factor. 


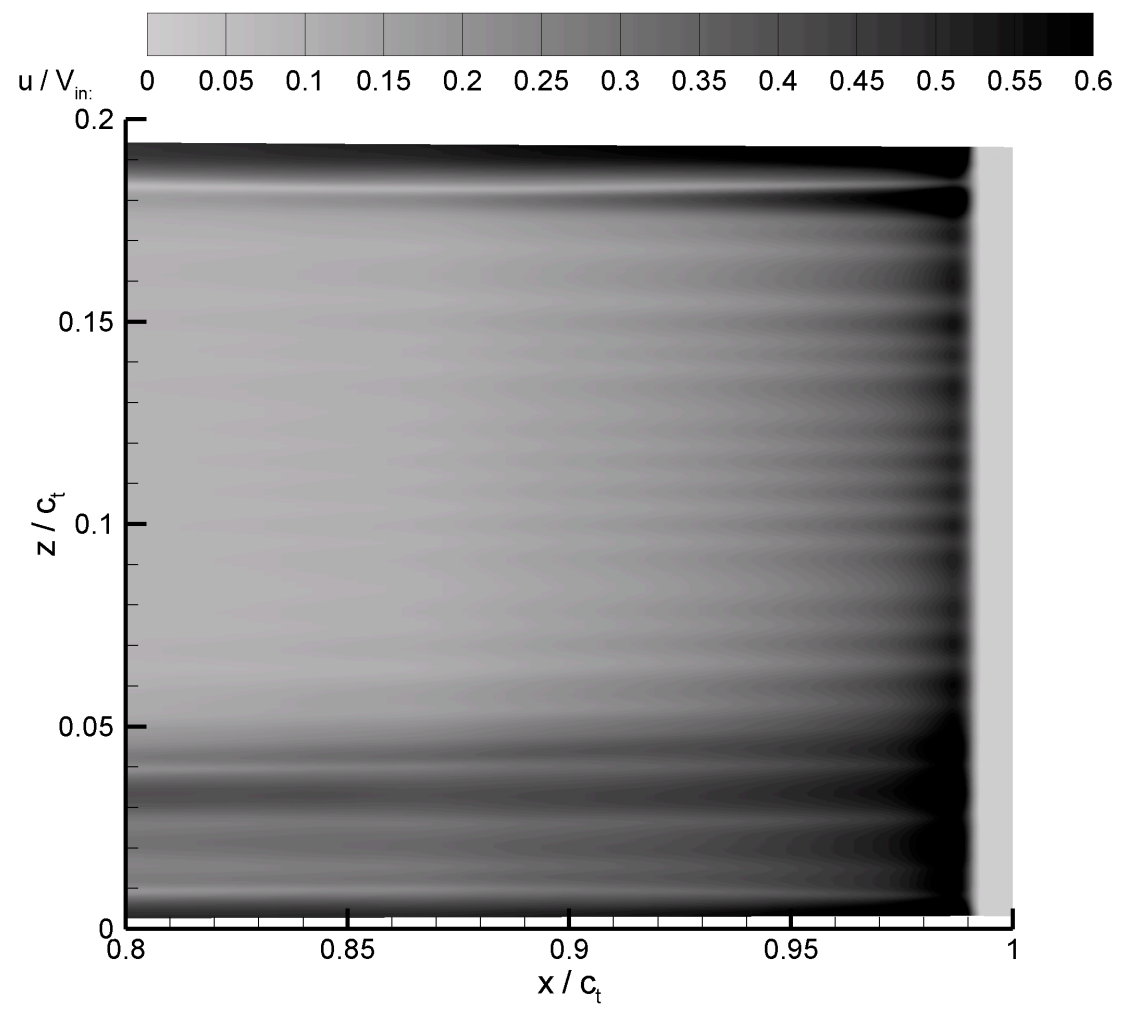

Fig. 6 Mean axial velocity near the pressure surface in Case 8. 


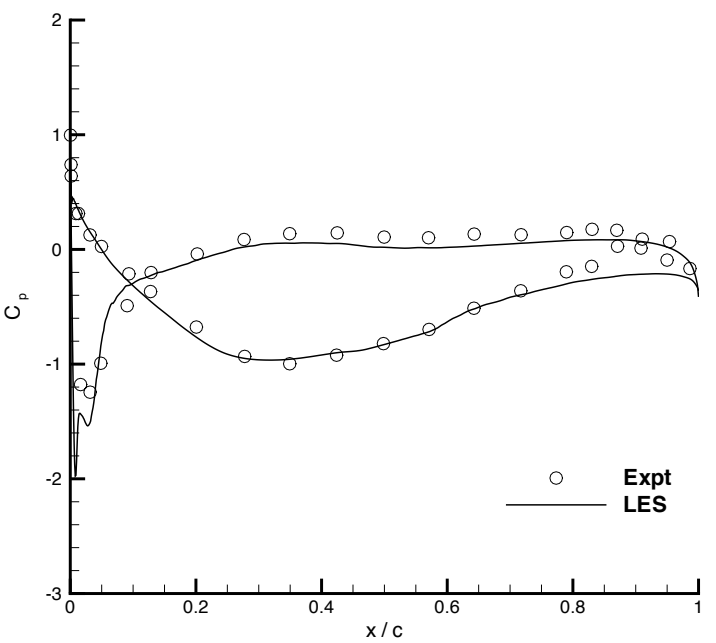

(a) Case 1

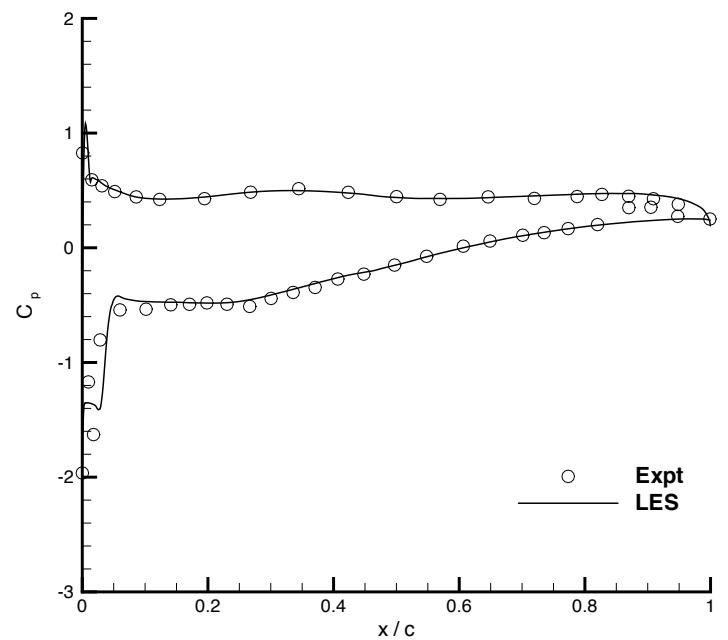

(b) Case 6

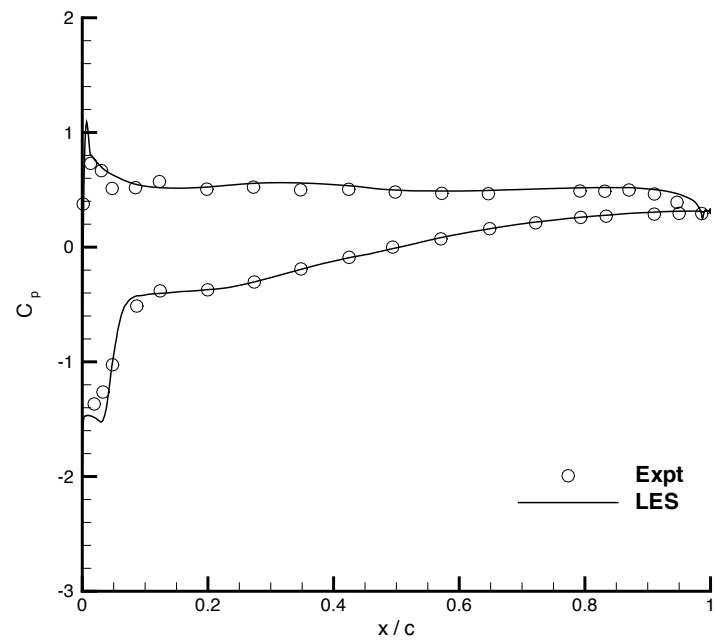

(c) Case 7

Fig. 7 Surface pressure distributions for Case (a) 1, (b) 6, and (c) 7 . 


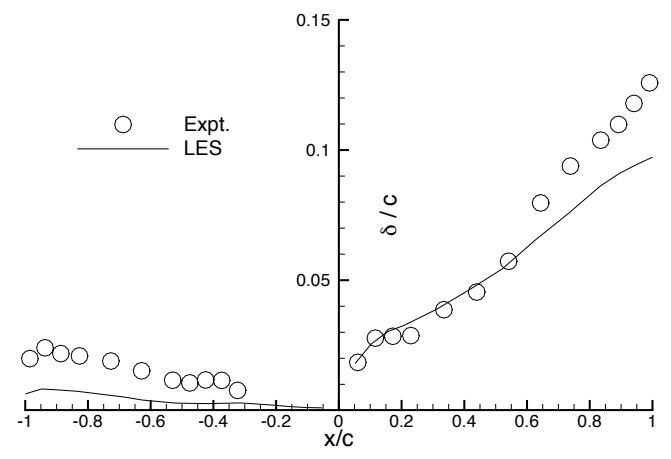

(a) Boundary Layer Thickness

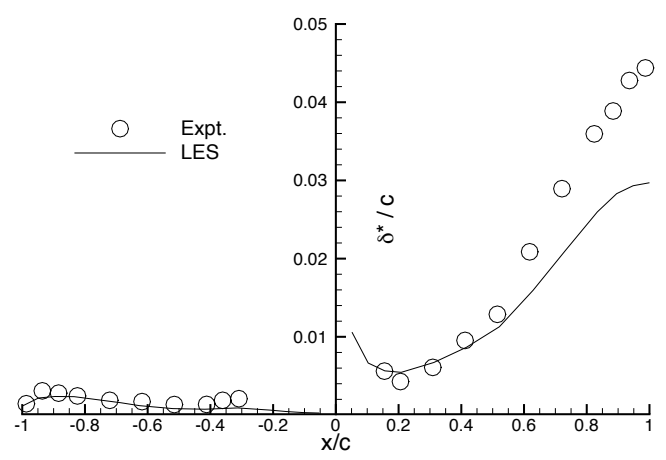

(b) Displacement Thickness

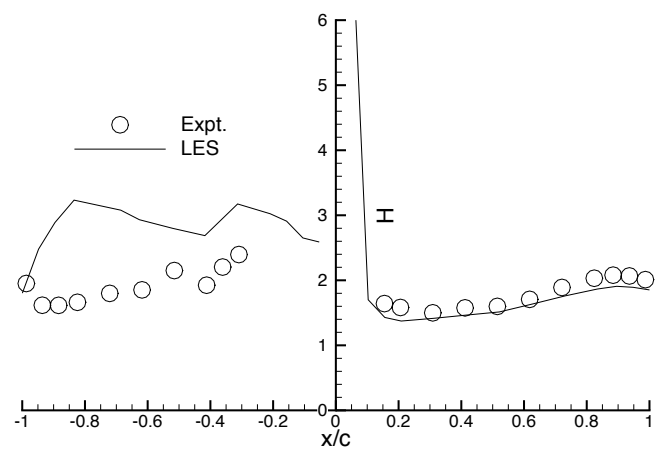

(c) Shape Factor

Fig. 8 Boundary layer properties for Case 7. (a) Boundary layer thickness, (b) Displacement thickness, (c) Shape factor. 


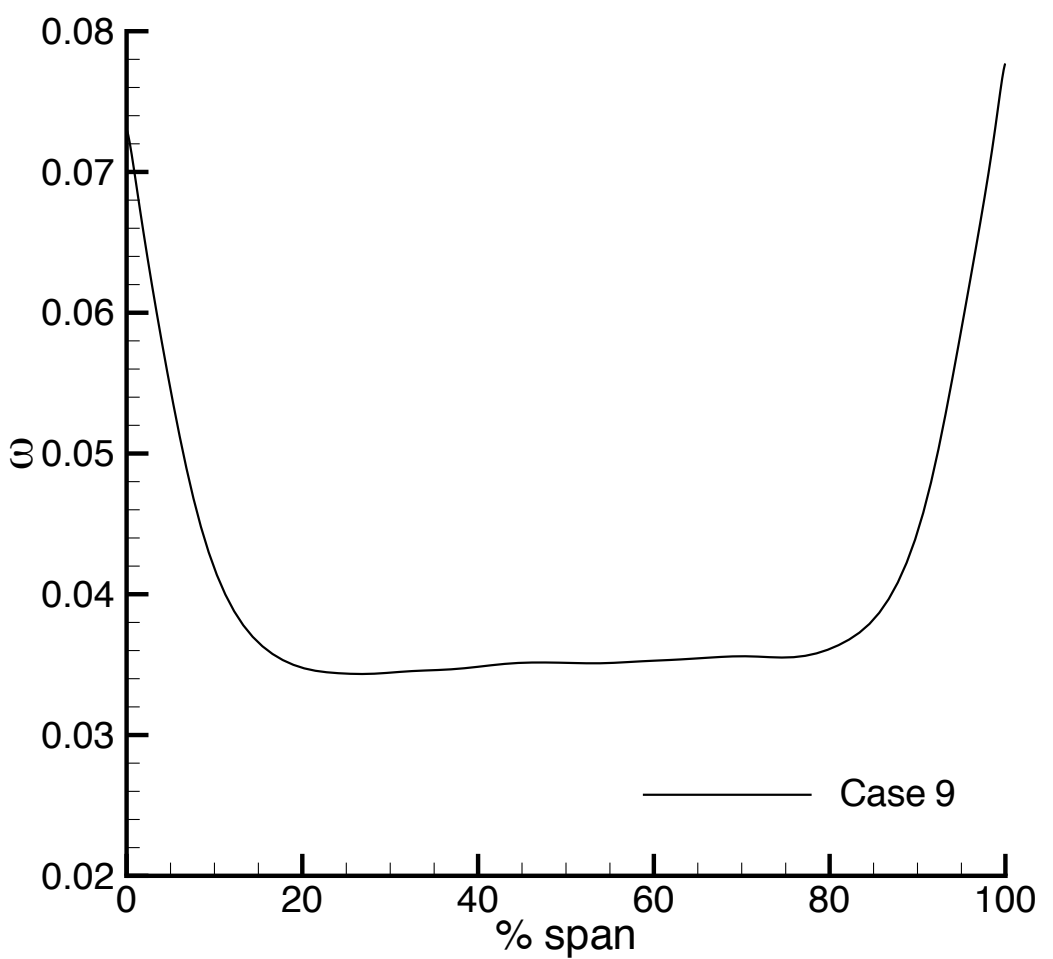

Fig. 9 Variation of loss with spanwise distance near the exit plane in Case 9. 


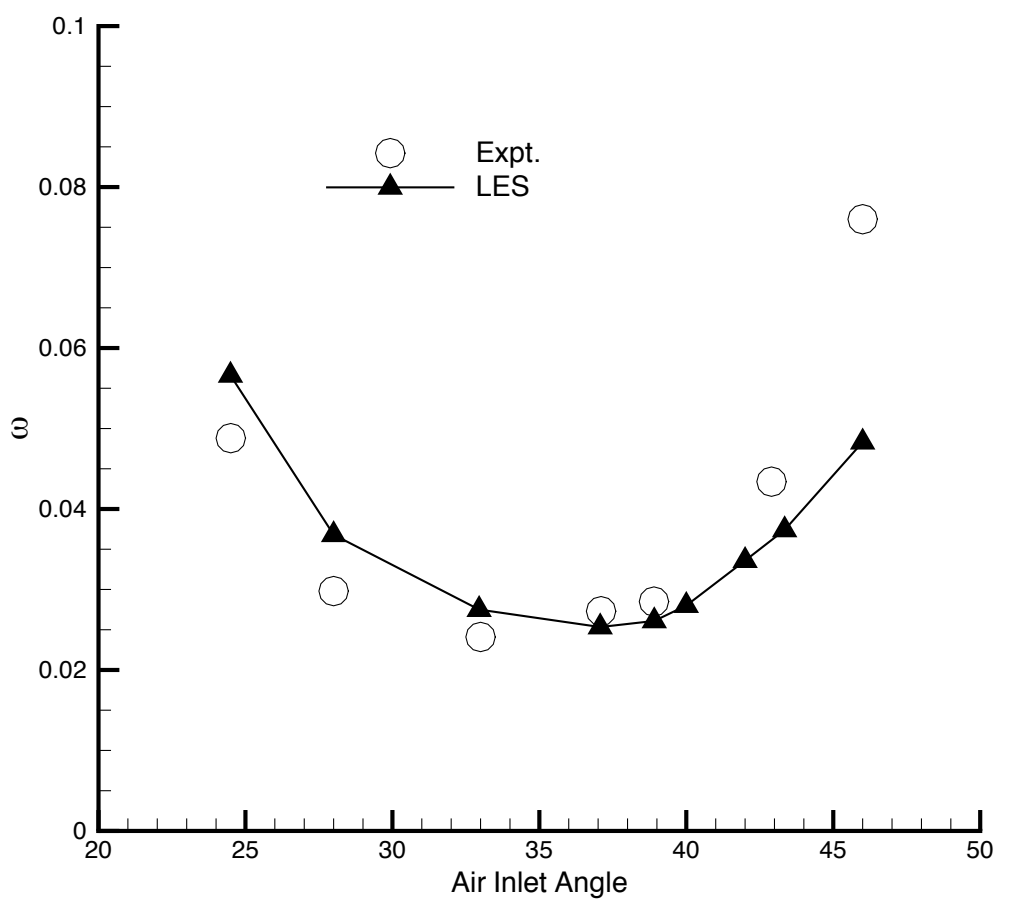

Fig. 10 Loss coefficient variation with flow inlet angle. 


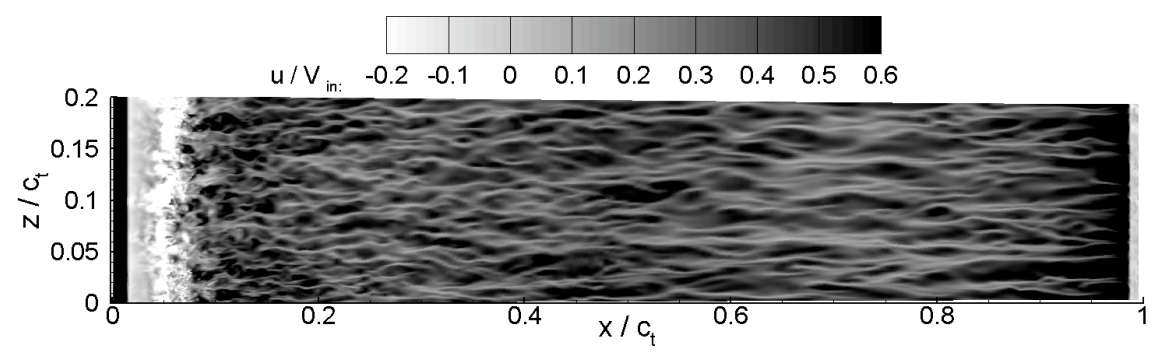

(a) Pressure side

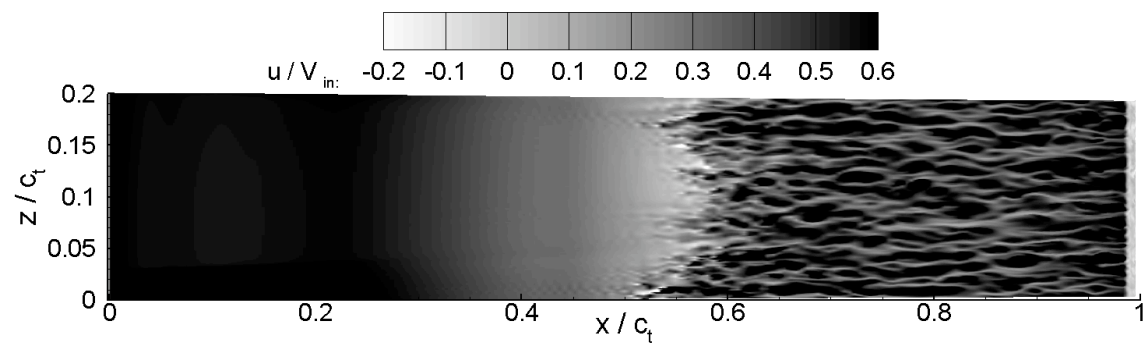

(b) Suction side

Fig. 11 Near-wall axial velocity distribution for Case $2, \beta=28^{\circ}$. (a) Pressure surface, (b) suction surface.

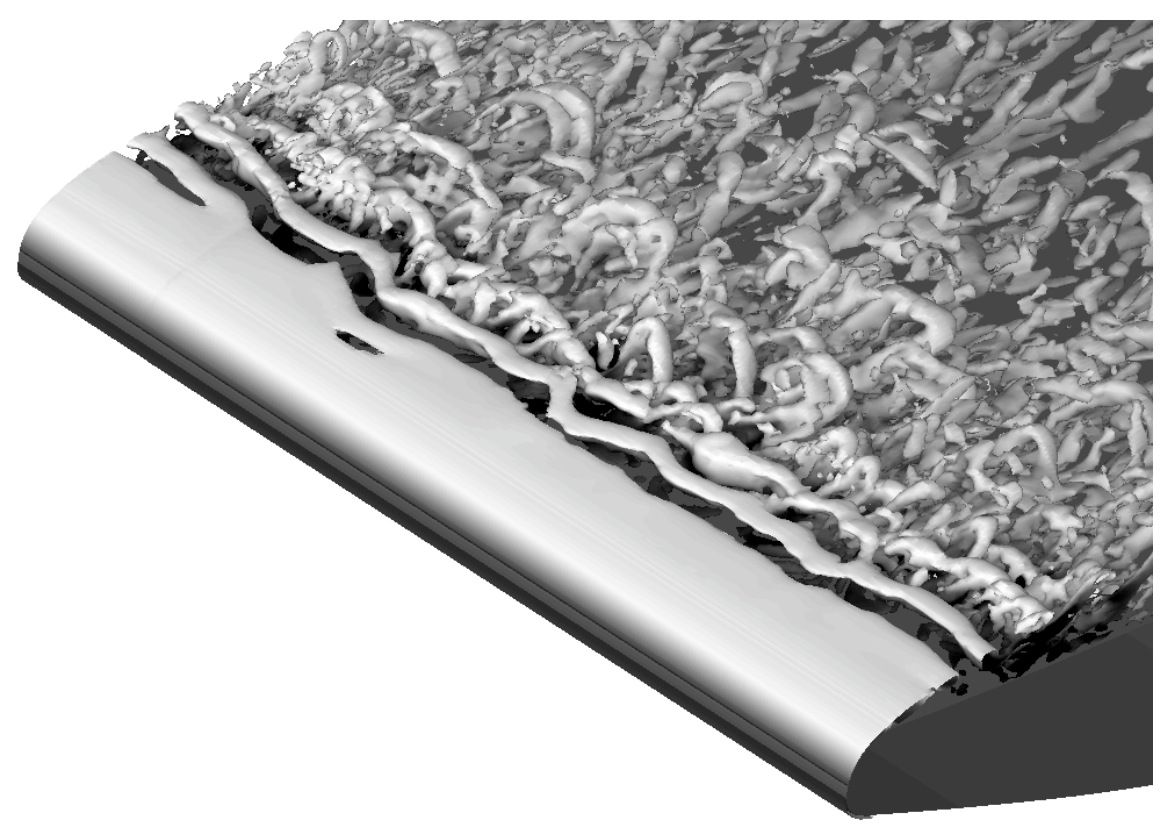

Fig. 12 Iso-surface of $Q-$ criterion at the leading edge in Case 2. Pressure surface on upper-side. 


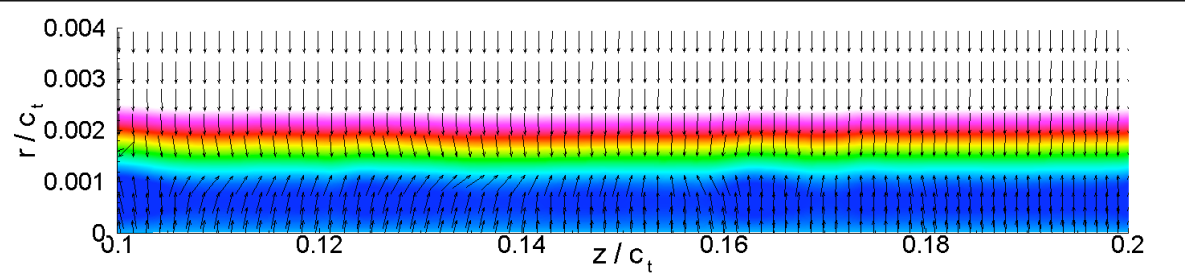

(a) $x / c_{t}=0.02$

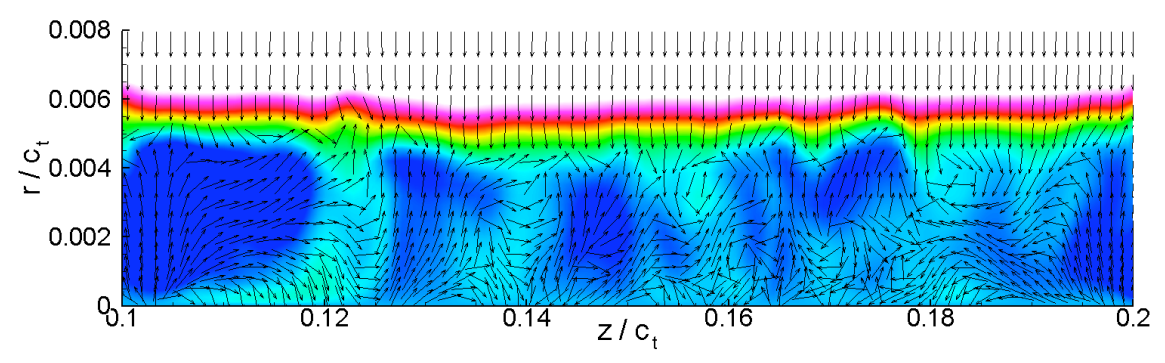

(b) $x / c_{t}=0.04$

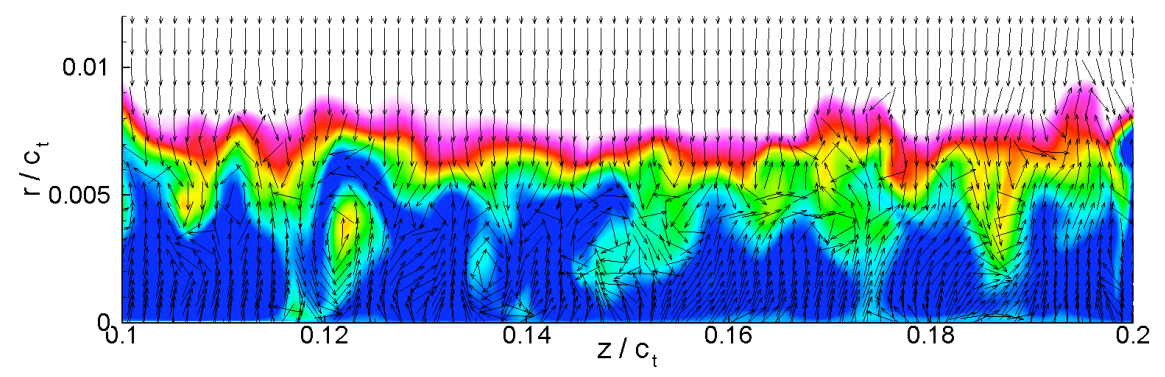

(c) $x / c_{t}=0.04$

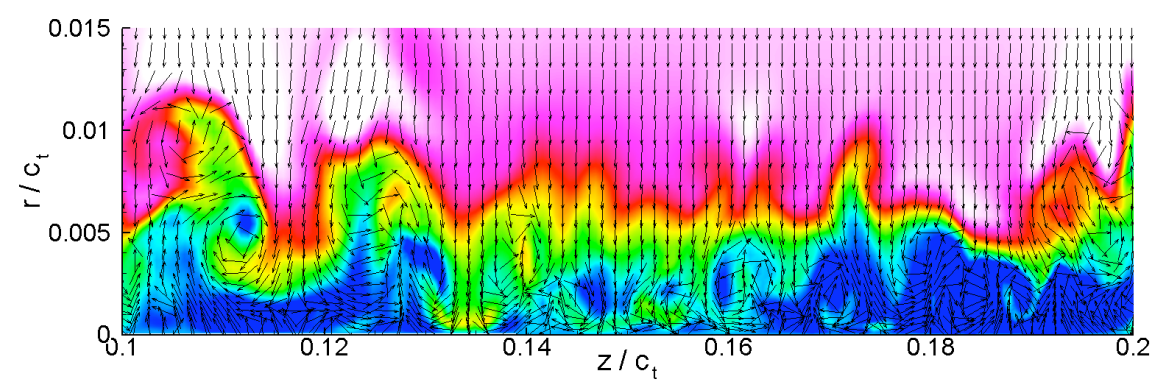

(d) $x / c_{t}=0.06$

Fig. 13 Cross-sections through the laminar separation bubble in Case 2. Colourmap as in Figure 14. (a) $x / c_{t}=0.0164$, (b) $x / c_{t}=0.0328, x / c_{t}=0.0492, x / c_{t}=0.0656$. 


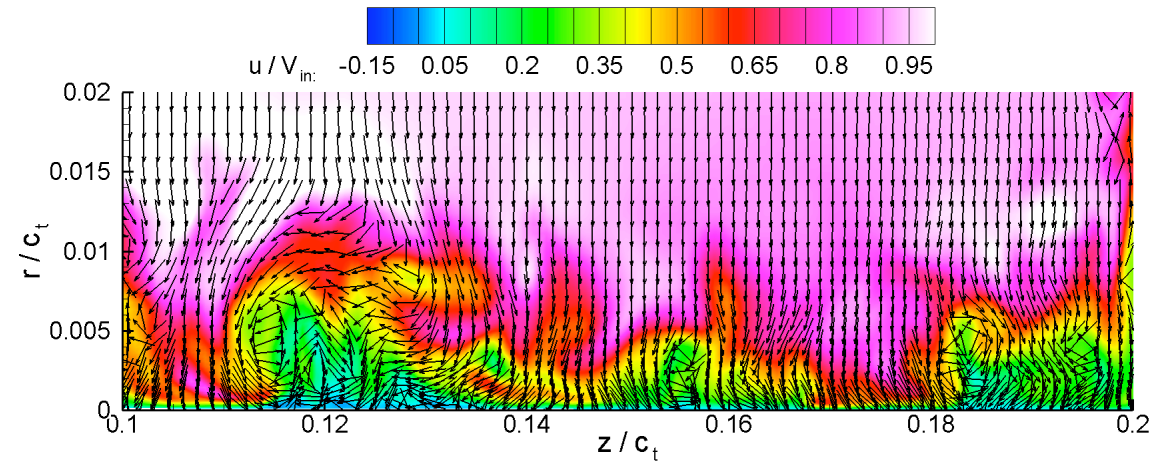

Fig. 14 Cross-section through the boundary layer of Case 2 at the approximate flow reattachment location, $x / c_{t}=0.0984$.

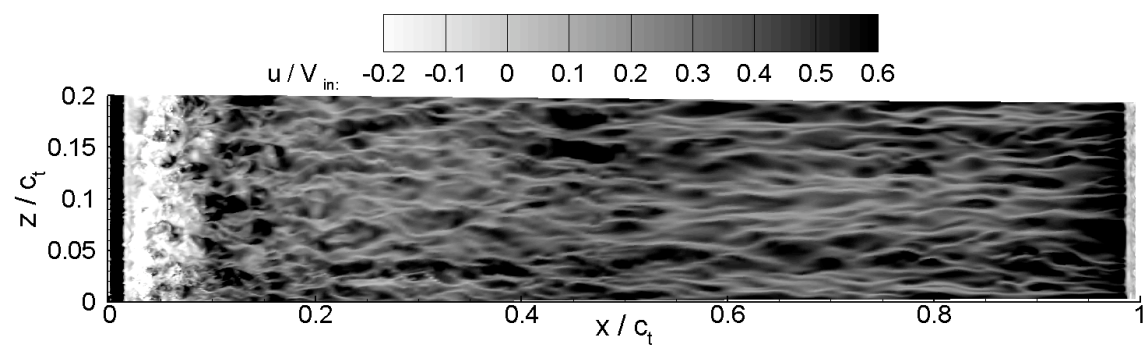

(a) Pressure side

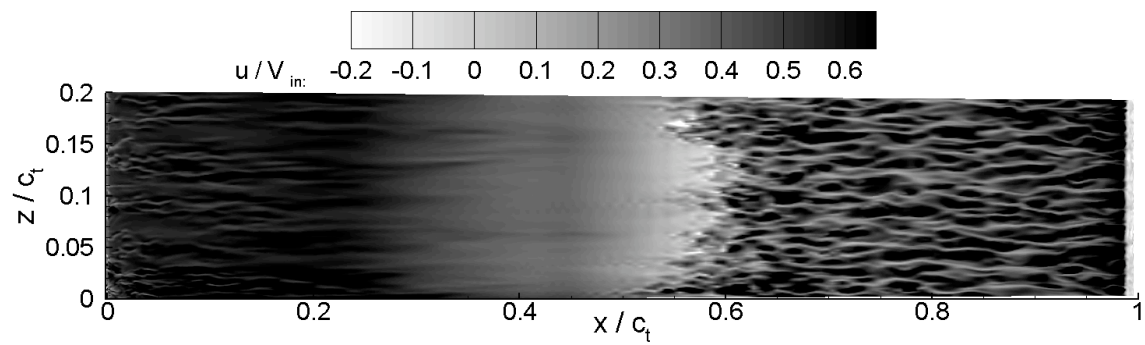

(b) Suction side

Fig. 15 Near-wall axial velocity distribution for Case $1, \beta=24.49^{\circ}$. (a) Pressure surface, (b) suction surface. 


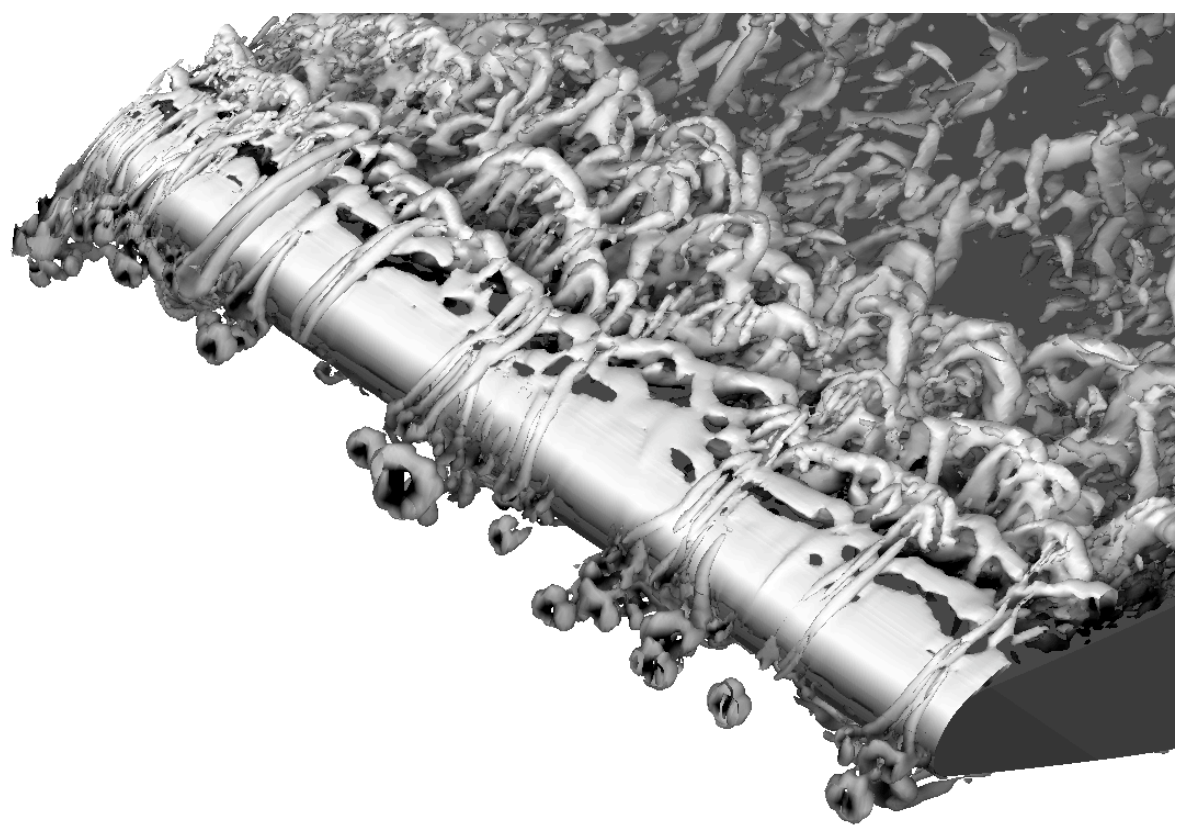

Fig. 16 Iso-surface of $Q>0$ at the leading edge in Case 1. Pressure surface on upper-side. 


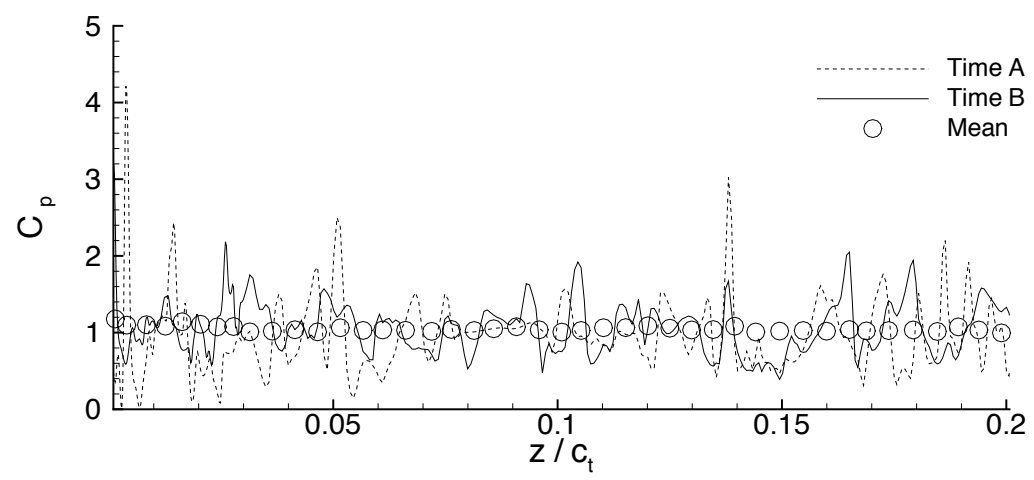

(a) Case 1

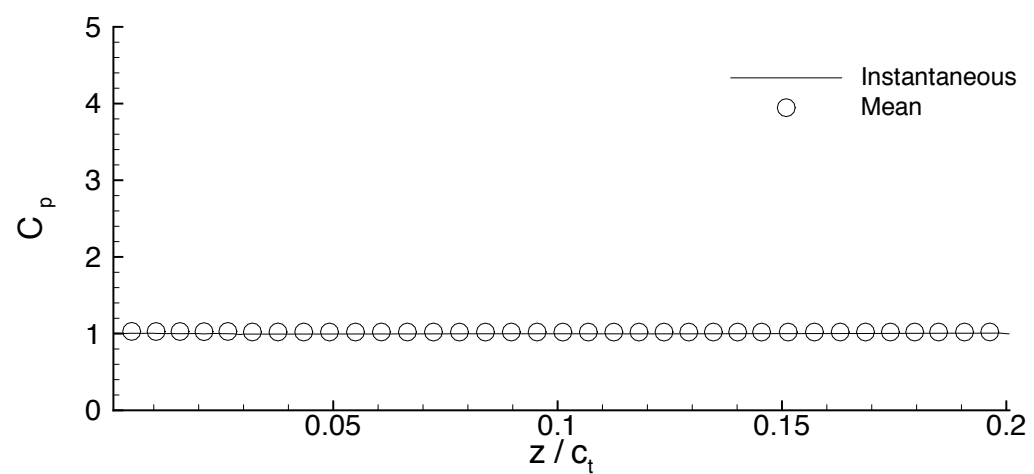

(b) Case 2

Fig. 17 Surface pressure coefficient distribution along the span of the blade at the location of the mean stagnation line in (a) Case 1, (b) Case 2 


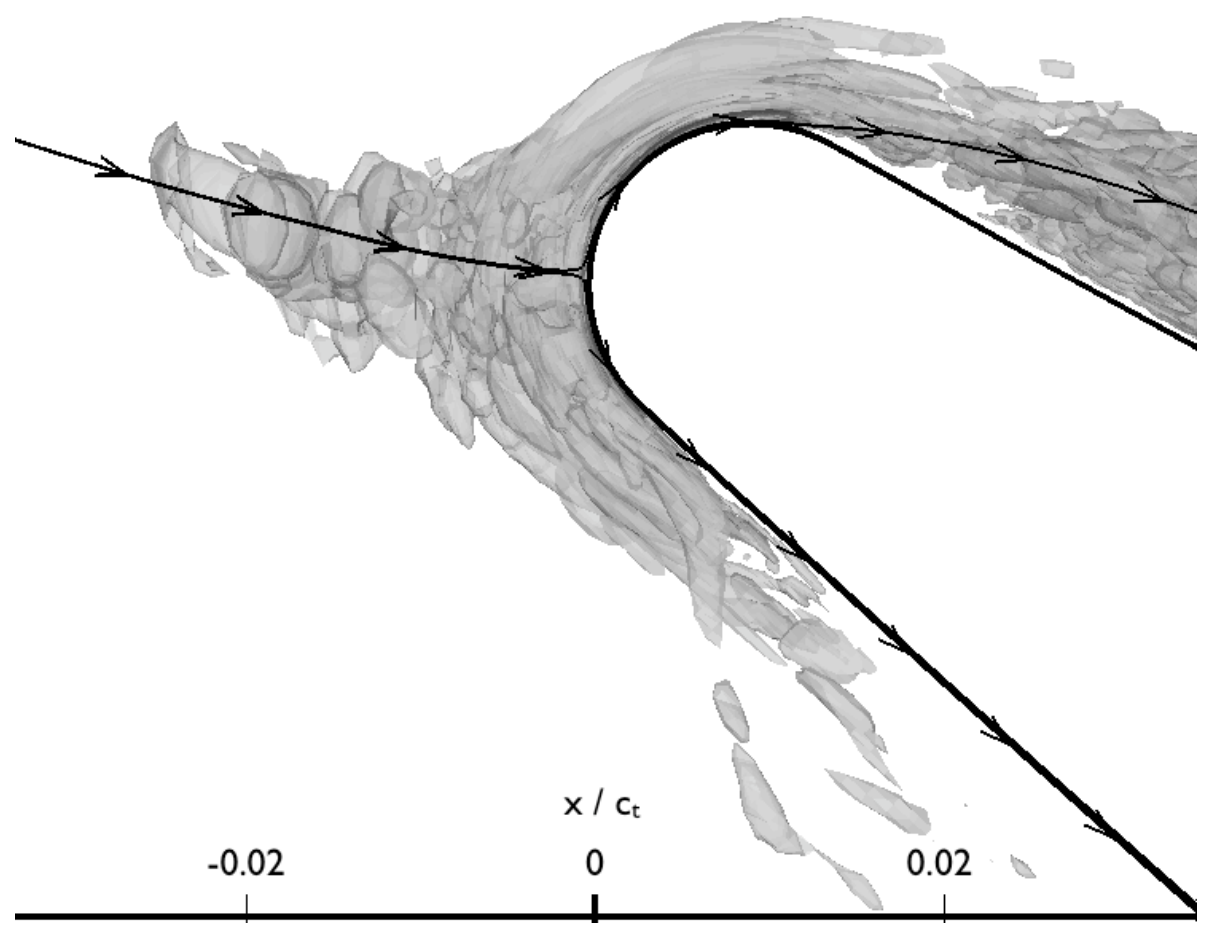

Fig. 18 Q-criterion iso-surface viewed with mean streamlines showing stagnation point at leading edge of blade in Case 1. 


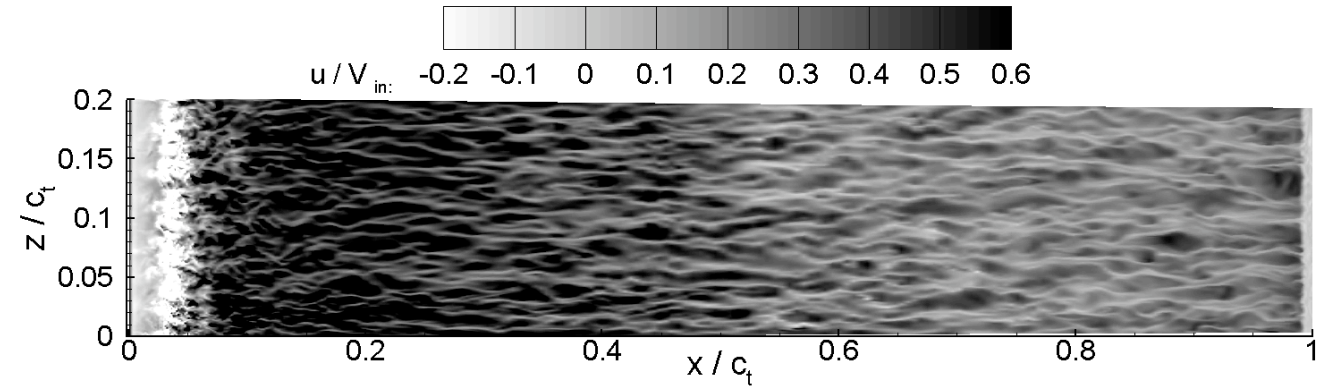

Fig. 19 Near-wall axial velocity distribution on the suction-surface in Case $9, \beta=43.4^{\circ}$.

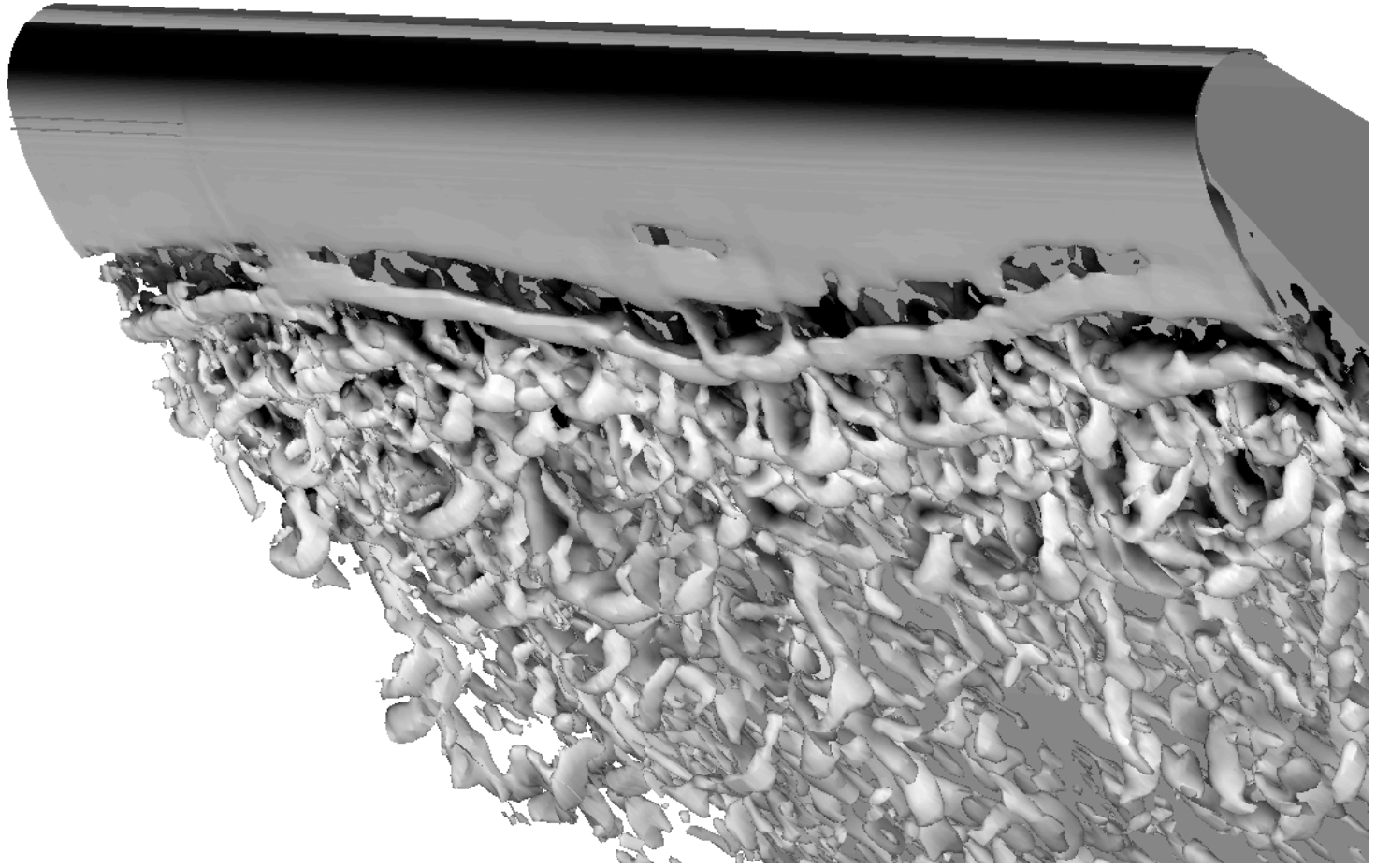

Fig. 20 Iso-surface of $Q-$ criterion at the leading edge in Case 9. Suction surface on under-side. 